\title{
State Changes Rapidly Modulate Cortical Neuronal Responsiveness
}

\author{
Andrea Hasenstaub, ${ }^{\star}$ Robert N. S. Sachdev, ${ }^{\star}$ and David A. McCormick \\ Department of Neurobiology, Kavli Institute of Neuroscience, Yale University School of Medicine, New Haven, Connecticut 06510
}

The responsiveness of cortical neurons is strongly and rapidly influenced by changes in the level of local network activity. In rodent somatosensory cortex, increases in network activity increase neuronal responsiveness to the intracellular injection of brief conductance stimuli but paradoxically decrease responsiveness to brief whisker deflections. However, whisker stimulation frequently evokes longlasting changes in the level of local circuit activity. The ability of stimuli to successfully evoke prolonged increases in circuit activity is associated with both an increase in the amount of conductance evoked by a whisker stimulus and an increase in action potential responsiveness to whisker stimulation. In addition, brief whisker stimuli presented during periods of high network activity evoke postsynaptic potentials containing a greater proportion of inhibition, consistent with an increased efficiency in the activation of inhibitory mechanisms during the Up state. In contrast, during prolonged and variable whisker stimulation, increased network activity is associated with an increase in overall responsiveness, dynamic range, output gain, and correlation between action potential response and speed of whisker movement. We conclude that stimulus-evoked or spontaneous alterations in cortical state can influence neuronal responsiveness in a complex manner, resulting in large changes in which, and how, sensory stimuli are represented.

Key words: background activity; Up states; adaptation; cortex; intracellular; network; noise; response; vibrissa (whisker)

\section{Introduction}

Cortical neurons are densely interconnected and tonically active, resulting in fluctuating barrages of synaptic activity that can profoundly control the cell's integrative and electrophysiological properties, the amplitude and time course of synaptic inputs, and the timing and probability of spiking. The slow oscillation, a recurrently generated cortical rhythm observed during slowwave sleep, under anesthesia, and in cortical slices in vitro, consists of spontaneously alternating periods of network activity and inactivity (Metherate and Ashe, 1993; Steriade et al., 1993; Cowan and Wilson, 1994; Sanchez-Vives and McCormick, 2000). During the active ("Up") state of the slow oscillation, cortical neurons fire at $0.1-25 \mathrm{~Hz}$ and are depolarized $5-25 \mathrm{mV}$ by barrages of balanced inhibitory and excitatory synaptic input, whereas during the inactive ("Down") state, cortical neurons are inactive and relatively hyperpolarized. The variable recurrent activity of the Up state is a model for natural fluctuations in the level of network activity during waking (Steriade et al., 2001; Crochet and Petersen, 2006). This ongoing network activity strongly facilitates action potential responsiveness to the intracellular injection of

Received Feb. 12, 2007; revised June 27, 2007; accepted July 12, 2007.

This work was supported by the National Institutes of Health, Howard Hughes Medical Institute, and the Kavli Center for Neuroscience.

${ }^{*}$ A.H. and R.N.S.S. contributed equally to this work.

Correspondence should be addressed to David A. McCormick, Department of Neurobiology, Kavli Institute of Neuroscience, Yale University School of Medicine, 333 Cedar Street, New Haven, CT 06510. E-mail: david.mccormick@yale.edu.

A. Hasenstaub's present address: Crick-Jacobs Center for Theoretical and Computational Neuroscience, Salk Institute for Biological Studies, 10010 North Torrey Pines Road, La Jolla, CA 92037.

DOI:10.1523/JNEUROSCI.2184-07.2007

Copyright $\odot 2007$ Society for Neuroscience $\quad$ 0270-6474/07/279607-16\$15.00/0 currents and to the presentation of visual stimuli, as well as the activation of both natural and intracellularly injected (synthetic) synaptic potentials (Arieli et al., 1995; Timofeev et al., 1996; Azouz and Gray, 1999; McCormick et al., 2003; Shu et al., 2003b; Haider et al., 2006). This facilitation results from networkinduced depolarization, which enhances neuronal responsiveness to a wide range of inputs, and increases in voltage fluctuations, which preferentially increase responsiveness to weak inputs (Shu et al., 2003b).

In apparent contradiction to these findings, the action potential responsiveness of rat primary somatosensory ("barrel") cortical neurons to brief whisker deflections is reduced during the Up state (Petersen et al., 2003; Sachdev et al., 2004). A variety of factors have been proposed, but not proven, to account for this decrease in responsiveness, including the reduction in driving force on excitatory synaptic potentials and increase in driving force on inhibitory synaptic potentials associated with depolarization, shunting associated with increased membrane conductance, depolarization-induced increases in spike threshold, increased recruitment of inhibitory mechanisms during periods of increased network activity, and short-term synaptic depression (Castro-Alamancos and Oldford, 2002; Petersen et al., 2003; Sachdev et al., 2004).

During normal behavior, whiskers may contact surfaces continuously and/or irregularly for tens or hundreds of milliseconds at a time (Sachdev et al., 2001, 2002; Knutsen et al., 2006), causing the whiskers to vibrate in a complex manner (Hartmann et al., 2003; Neimark et al., 2003; Hipp et al., 2006). The effect of background activity on cortical responsiveness to such patterned, prolonged vibrissal stimulation is not known. Through a combination of facilitation of responsiveness to weak inputs, increased 
spike timing variability, and reduction in response reliability, cortical background activity can increase the correlation of neuronal spike activity to fluctuations in synaptic inputs (Shu et al., 2003b). These same mechanisms might operate to increase the ability of cortical neurons to represent prolonged, fluctuating whisker stimuli in their action potential output.

Here we use Up and Down states of the slow oscillation in rat primary somatosensory cortex in vivo to address the mechanisms by which changes in the level of local network activity can alter neuronal responsiveness to vibrissal stimulation and how these changes vary as a function of the temporal characteristics of the stimulation.

\section{Materials and Methods \\ Surgery}

Adult male Sprague Dawley rats weighing 270-495 g were used in these experiments. Animals were anesthetized with urethane $(20 \%$ w:v; 1.5 $\mathrm{g} / \mathrm{kg}$ body weight, i.p.) and supplemented with ketamine/xylazine (35 $\mathrm{mg} / \mathrm{kg}$ ketamine and $7 \mathrm{mg} / \mathrm{kg}$ xylazine, i.m., hourly), respectively. A craniotomy was made over the somatosensory barrel cortex, and the cisterna magna was drained to relieve intracranial pressure.

\section{Recording methods}

Electrophysiology. Intracellular recordings were performed using conventional sharp electrodes, pulled on a P-80 micropipette puller (Sutter Instruments, Novato, CA) from medium-walled glass capillaries (1BF150; World Precision Instruments, Sarasota, FL), and electrode tips were broken back to give final resistances of 35-90 M $\Omega$. Except where otherwise specified, electrodes contained $1 \mathrm{~m}$ potassium acetate (KAc) and $1-2 \%$ biocytin. For voltage-clamp experiments, electrodes contained $50 \mathrm{~mm}$ $\mathrm{N}$-(2,6-dimethylphenylcarbamoylmethyl)triethylammonium bromide (QX-314) and $2 \mathrm{~m}$ cesium acetate (CsAc), which blocks voltage-dependent $\mathrm{Na}^{+}$currents and reduces $\mathrm{K}^{+}$currents, the hyperpolarization-activated cation current (h-current), and $\mathrm{GABA}_{\mathrm{B}}$-mediated synaptic potentials. Intracellular signals were amplified with an Axoclamp-2B amplifier (Molecular Devices, Foster City, CA) in either current-clamp or single-electrode voltage-clamp mode. The recorded signals were acquired, digitized, and analyzed at $20 \mathrm{kHz}$ with a Spike2 system and software (Cambridge Electronic Design, Cambridge, UK). Capacitance compensation, DC offset, and bridge balance were monitored and carefully adjusted as the electrode was advanced into the brain. During single-electrode voltage clamp, the headstage output of the Axoclamp-2B amplifier was continuously monitored to ensure adequate settling time.

Cell selection. Intracellular recordings were accepted if they showed a stable membrane potential below $-55 \mathrm{mV}$ at rest, action potentials that crossed $0 \mathrm{mV}$, and an input resistance larger than $20 \mathrm{M} \Omega$. For singleelectrode voltage clamp, cells had to have zero crossing action potentials immediately after impalement, membrane potential had to be below $-55 \mathrm{mV}$ at rest, the sampling rate had to be at least $2.8 \mathrm{kHz}$, cells had to have an input resistance of $20 \mathrm{M} \Omega$, and stable recordings had to be obtained at five different holding potentials (including potentials more depolarized and more hyperpolarized than reversal) including 30-60 trials and 20-30 Up states at each membrane potential.

Electrophysiologically characterized regular-spiking, fast-spiking, and intrinsic-bursting neurons were combined in our analyses, except where specified. Cell classification was performed according to established procedures (McCormick et al., 1985; Nowak et al., 2003). A subset of neurons were stained and recovered: this subset included two neurons with pyramidal morphology whose cell bodies were located in layers $2 / 3$, nine neurons with pyramidal morphology whose cell bodies were located in layer 5 , and one neuron with basket morphology whose cell body was located in layer 4. Both barrel-column and septal-column neurons were recovered and combined in this analysis.

\section{Stimulation}

Identification of principal whisker. All whiskers were trimmed to $\sim 1 \mathrm{~cm}$, and a $1.5 \mathrm{~mm}$ glass pipette attached to a piezoelectric stimulator (with feedback and waveform output; E-651; Physik Instrumente, Karlsruhe,
Germany) was positioned at the cut end of a single whisker. The whisker to be stimulated was chosen by first identifying the whisker evoking the best response in the nearby extracellular electrode. All whiskers adjacent to the putative principal whisker were stimulated, to confirm that their stimulation resulted in a weaker and longer-latency response.

Whisker stimulation. Whisker stimuli were generated in one of two ways. For most experiments, the stimulus used was a trapezoidal rampand-hold stimulus with a fixed ramp on time (5-10 ms), hold time (100 $\mathrm{ms})$, and slow (100 ms) ramp off delivered once every $500 \mathrm{~ms}$. The maximum deflection (and thus the rate of deflection) was randomly varied from an amplitude of 0 (i.e., no stimulus, or control) to $2 \mathrm{~mm}$, the maximum deliverable in closed-loop configuration, with accurate waveform output. Stimulus amplitude was randomized to prevent orderspecific effects.

For investigation of the effect of cortical state on responsiveness to prolonged, fluctuating whisker deflections, the whisker stimulus was generated using an Ornstein-Uhlenbeck process (Uhlenbeck and Ornstein, 1930; Fellous et al., 2003) to produce whisker deflections with time constants of 5 or $40 \mathrm{~ms}$. When using fluctuating stimuli, the optimal stimulus direction and parameters were first selected by examining online spike-triggered averages (for randomly fluctuating noisy stimuli) and peristimulus time histograms (PSTHs) (for repeating noisy stimuli) of the four ordinal stimulus directions. For reverse-correlation analyses, stimuli with $5 \mathrm{~ms}$ time constants were used. For measurement of the correlation between whisker stimulation and spike output patterns, stimuli with time constants of 5 and $40 \mathrm{~ms}$ did not yield substantially different results and were combined in our analysis, after correction for stimulus autocorrelation.

Dynamic clamp. Excitatory postsynaptic conductances were simulated and injected using our dynamic clamp system. Our dynamic clamp experiments were implemented in software developed by combining realtime Linux (Dorval et al., 2001), the freely available COMEDI data acquisition device control libraries (www.comedi.org), and model-specific C code; this software was used to control a DAP-5216a data acquisition board (Microstar Laboratory, Bellevue, WA). Conductance calculations were performed at a rate of $10 \mathrm{kHz}$. The time course of the synthetic conductances was defined by a kinetic model of synaptic transmission including only glutamate AMPA receptors (Pare et al., 1998). Artificial excitatory postsynaptic conductances were injected at $5 \mathrm{~Hz}$, and their amplitudes were randomly generated (from a peak amplitude minimum of 0 or $2 \mathrm{nS}$ to a peak amplitude maximum sufficient to reliably evoke action potentials regardless of neuronal state) so as to prevent orderspecific effects.

\section{Analysis}

Presence of Up and Down states. Up states were taken to be present in an intracellular or extracellular recording when the recording showed two distinct, stable (periods lasting $100 \mathrm{~ms}$ or longer), spontaneously alternating activity levels (see Fig. $1 \mathrm{~A}$ ). In an extracellular single- or multipleunit (MU) recording, these corresponded to periods of higher spike rate in the recorded neuron or group of neurons alternating with periods of lower [although not necessarily zero (Compte et al., 2003)] spike rate (see Fig. $1 A$, top), whereas in an intracellular recording, these corresponded to periods of intracellular depolarization, often including action potential generation, alternating with periods of intracellular hyperpolarization (see Fig. $1 \mathrm{~A}$, bottom). To determine whether a given stimulus was presented during the Up or the Down state, the multiple-unit trace (for the voltage-clamp recordings in Fig. 8) or membrane potential trace (for Figs. 3-7, 9-11) was first filtered to yield an outline of the increases and decreases in activity associated with changes in cortical state (see Fig. 1 B). Multiple-unit recordings were rectified, median filtered, and smoothed (see Fig. $1 B$, top), whereas voltage traces were simply median filtered and smoothed (see Fig. $1 B$, bottom). A histogram of the resulting activity trace [filtered MU or membrane potential $\left(V_{\mathrm{m}}\right)$ ] was constructed (see Fig. 1C). This histogram was clearly bimodal, corresponding to the Down and $\mathrm{Up}$ states (see Fig. 1C). The lower threshold was placed just above the upper limit of the membrane potentials or MU activity for the Down state, at 5-10 percentiles below the trough in the activity histogram, whereas the upper threshold was placed just below the lower limit of the 
Up state, at 5-10 percentiles above the trough in the activity histogram (see Fig. 1C,D). The filtered activity traces closely tracked the unfiltered traces (see Fig. $1 D$ ). In addition, the activity traces obtained from the multiple-unit and membrane potential recordings closely tracked one another (see Fig. 1C,E).

Cortical state at time of stimulus delivery. Cortical state at the time of stimulus delivery was classified by comparing the activity trace to the upper and lower thresholds (see Fig. 1C,D) for the $50 \mathrm{~ms}$ before stimulus delivery, time shifted by $0-15 \mathrm{~ms}$ to compensate for cortical response latency (see Fig. 2). In Figures 3-8, discrete whisker stimuli were classified as having been delivered during the Down state if, for the $50 \mathrm{~ms}$ before the stimulus, the trace remained below the lower threshold (see Fig. $2 \mathrm{Ba}$ ). Stimuli were classified as having been delivered during the Up state if, for the $50 \mathrm{~ms}$ before the stimulus, the trace passed at least once above the higher threshold and remained elevated above the lower threshold (see Fig. $2 \mathrm{Bc}$ ). Approximately three-quarters of stimuli were classified into one of these two sets. The remaining stimuli (see Fig. $2 \mathrm{Bb}$ ) immediately followed a spontaneous Up state initiation (see Fig. 2Ca), a spontaneous Up state termination (see Fig. $2 C c$ ), or an unclassifiable period of the recording (see Fig. $2 \mathrm{Cb}$ ). These remaining stimuli were not included in further analyses. This was the only classification method used for the analyses in Figures 3-5.

Identification of state transitions. In Figures 6-8, discrete whisker stimuli were first classified according to whether they were delivered during the Up or the Down state, as described above (see Fig. 2). They were then subclassified according to the activity trace during a 30-50 ms window beginning $50 \mathrm{~ms}$ after stimulus onset, time shifted by $0-15 \mathrm{~ms}$ to compensate for system latency. Stimuli delivered during the Up state were classified as having terminated the Up state if the activity trace remained below the lower threshold during the poststimulus time window (see Fig. $7 \mathrm{Db}$ ). Stimuli delivered during the Down state were classified as having evoked an Up state if, during the poststimulus window, the trace passed above the higher activity threshold and remained above the lower activity threshold (see Fig. 7Eb).

The durations of the poststimulus PSPs were calculated by measuring the time from the initial rise of membrane potential after stimulation until the membrane potential fell below the lower threshold (i.e., returned to baseline) (see supplemental Fig. 1, available at www.jneurosci.org as supplemental material). PSP duration histograms were constructed and analyzed for bimodality using an implementation of Hartigan's dip test (Hartigan and Hartigan, 1986) based on public domain code provided by F. Mechler and D. Ringach (University of California, Los Angeles, Los Angeles, CA).

The times of Down-to-Up and Up-to-Down state transitions were identified by examining the intracellularly recorded membrane potential. A crossing of the lower threshold was taken to be an Up state initiation if before the crossing, the voltage remained below the lower threshold for at least $50 \mathrm{~ms}$, and if after the crossing, the voltage rose above and remained above the higher threshold for at least $100 \mathrm{~ms}$ (see Fig. $6 \mathrm{~Eb}$ ). A crossing of the upper threshold was taken to be a Down state initiation if, before the crossing, the voltage had remained above the higher threshold for at least $100 \mathrm{~ms}$, and if, after the crossing, the voltage fell below the lower threshold and remained below the lower threshold for at least 100 $\mathrm{ms}$ (see Fig. $6 \mathrm{Db}$ ). Histograms of transition probability synchronized to stimulus onset were constructed (not shown), and within-cell significance of observing a transition was measured by estimating the probability that the $0-50 \mathrm{~ms}$ bin was drawn from the same distribution as the -50 to $0 \mathrm{~ms},-100$ to $-50 \mathrm{~ms}$, etc. bins, calculated using a $\chi^{2}$ goodnessof-fit test. The stimulus-evoked change in transition probability was taken to be the baseline-subtracted probability of observing a state transition in each $50 \mathrm{~ms}$ period after a stimulus. The across-cell significance of increases in transition probability after a stimulus was calculated using a Wilcoxon signed rank test.

Continuous stimulation. In Figures 9-11, whisker stimuli were delivered continuously rather than discretely. The minimum response latency was determined from PSTHs of the spike response to discrete stimuli, and a spike was classified as an Up-evoked spike if a discrete whisker stimulus delivered at time $t=$ (spike time - system latency) would have been classified as an Up-delivered stimulus (see above and Figs. 1, 2), and as a Down-evoked spike if a discrete whisker stimulus delivered at time $t$ $=($ spike time - system latency $)$ would have been classified as a Downdelivered stimulus.

Input-output relationships. The input-output relationships in Figures $3-5$ were measured by counting the number of spikes generated within the first $50 \mathrm{~ms}$ after stimuli of a particular size and dividing this spike count by the number of stimuli of that size presented during the entire experiment, for Up and Down states separately. This $50 \mathrm{~ms}$ window was chosen because plots of the PSTH of spikes generated after brief whisker deflections showed this to be the maximum window of time during which a reliable increase in spike rate above both Down state and Up state baseline was observed (see Fig. 2; supplemental Fig. 1, available at www. jneurosci.org as supplemental material). The baseline spike count was calculated by measuring the mean spike count during the $50 \mathrm{~ms}$ before a stimulus, for Up and Down states separately. Baseline and stimulusevoked spike rates were measured by dividing this spike count by the duration of the time window over which they were counted. In Figure $3 D$, input-output curves were normalized to the maximum (nonbaseline-subtracted) spike rate: because the observed spike rate exhibited some saturation at strong stimulus sizes, the maximum spike rate did not always fall in the same bin, and no bin normalized to exactly $100 \%$. In Figure $4 C$, input-output curves were normalized on the $x$-axis (to compensate for the variety of input resistances of the recorded cells), to the stimulus size that evoked an additional spike on one-half of trials during the Up state.

Voltage clamp. The change in membrane conductance during the Up state, compared with the baseline conductance during the Down state, was measured using a modification of the method of Borg-Graham (Borg-Graham et al., 1998). At each of several different membrane potentials, postsynaptic currents occurring after a stimulus were measured multiple (10-30) times under single-electrode voltage clamp. The multiple recorded PSP currents were sorted according to whether the time of stimulus onset would be classified as an Up, Down, or Evoking stimulus, based on observation of a nearby multiple-unit electrode as described above. Current versus voltage plots were constructed for baseline $(-20$ to $0 \mathrm{~ms}$ before stimulus onset) and every $3 \mathrm{~ms}$ thereafter. The change in slope between the $I-V$ plot for the baseline and the $I-V$ plot at each time during the PSP, calculated using reduced major axis regression from measurements at voltages surrounding the observed reversal potential, was taken as the change in membrane conductance at that time during the PSP state. The point of crossing of the $I-V$ fits was taken as the reversal potential (for time points when the measured conductance was elevated above baseline). For the group data in Figure $8 C$, both conductance and reversal potential were measured at the time of peak conductance.

Kernel identification. To determine the stimulus feature that neurons responded to most reliably, autocorrelation-normalized spike-triggered averages of the whisker position, velocity, and speed (rectified velocity) were constructed for all spikes and for Up and Down spikes separately. Consistent with previous results (Pinto et al., 2000; Shoykhet et al., 2000; Arabzadeh et al., 2004), we found the most reliable relationship between action potential discharge probability and whisker speed (rectified velocity), rather than velocity or position (indeed, five of six cells examined exhibited no significant relationship between whisker position and spike output). We therefore confined our further investigations to the relationship between whisker speed and spike output, and we use "stimulus" as shorthand for "rectified whisker velocity" in subsequent analyses and discussion. The spatiotemporal receptive field (STRF) of each neuron was taken to be the autocorrelation-corrected spike-triggered average of whisker speed between 150 and $0 \mathrm{~ms}$ before action potential generation.

Scale and correlation. We quantified the relationship between the stimuli preceding individual spikes and the average prespike stimulus by measuring correlation and scale for each prespike stimulus (supplemental Fig. 3, available at www.jneurosci.org as supplemental material). The correlation (and significance of the correlation) between a particular prespike stimulus and the kernel was taken as Pearson's correlation coefficient (and its $p$ value) of the two vectors. The scale, or the amount by which the mean prespike stimulus would need to be stretched to best fit a particular prespike stimulus, was taken to be the slope of the linear fit of the particular prespike stimulus against the kernel. Spikes were then 
classified according to whether the correlation between the prespike stimulus and the kernel was not significant $(p<0.05)$, significantly positive, or significantly negative, and, among significantly correlated spikes, whether the scale was large or small. For each recording, the proportions of spikes falling into each group were calculated for Up and Down states separately.

Gain estimation. Estimation of the output gain in the Up and Down states was performed using a linear-nonlinear model (de Boer and Kuyper, 1968; Powers and Arnett, 1981; Theunissen and Miller, 1995; Ringach et al., 2002). Whisker speed was first convolved against the autocorrelation-normalized spiketriggered average of the whisker speed, producing a "likeness," or measurement of similarity of the stimulus to the estimated optimal stimulus (spike-triggered average of previous whisker speed) as a function of time. These likeness measurements were then grouped into several bins, from worst (corresponding to times when the prespike stimulus was anticorrelated with the average prespike stimulus) to best (corresponding to times when the prespike stimulus strongly resembled the average prespike stimulus). Each time point was then classified as Up or Down, depending on how a spike generated at that time would have been classified (see above). Finally, for Up and Down times separately, the number of time points (i.e., chances for spike generation) in each likeness bin was counted and compared with the number of spikes actually generated during these times, thus producing input-output curves in units of spikes/s. For each cell, gain was measured both as the absolute difference in firing rate between best and worst stimuli and as the percentage change in firing rate between best and worst stimuli.

\section{Results}

Up states in barrel cortex diminish responsiveness to brief whisker deflections

Intracellular recordings from neurons $(n=27)$ simultaneous with extracellular multiple-unit recordings in the primary somatosensory cortex of ketamine/ xylazine-anesthetized rats $(n=27)$ exhibited spontaneous, periodic Up states, characterized by depolarization, increased membrane potential variability, spike generation, and increased multiple-unit spike activity, interspersed with Down states, characterized by membrane hyperpolarization, the absence of spikes in the intracellularly recorded neuron, and reduced multiple-unit spike activity (Fig. 1). To examine the effects of changes in local network activity on the inputoutput relationships of cortical neurons, the principal whisker (defined as the whisker that evoked the shortest-latency, largestamplitude response in a PSTH) was stimulated with a randomamplitude trapezoidal ramp-and-hold whisker deflection once every $500 \mathrm{~ms}$. These stimuli were sorted according to the cortical state (Up or Down) just before stimulus onset (see Materials and Methods) (Figs. 1, 2, 3A). During the Up state, neurons exhibited

E
Raw Data

MU

$\mathrm{Vm}$

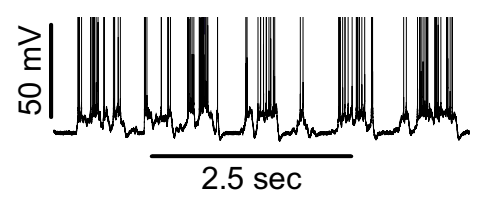

C

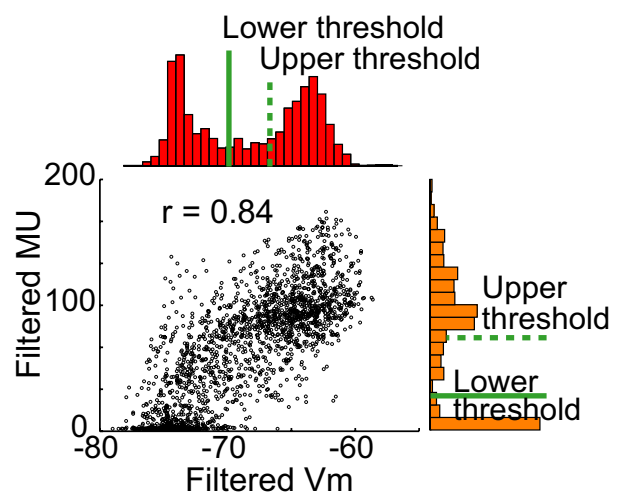

B Activity Traces

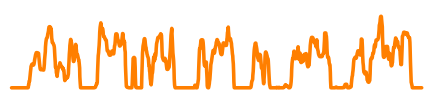

Filtered Vm
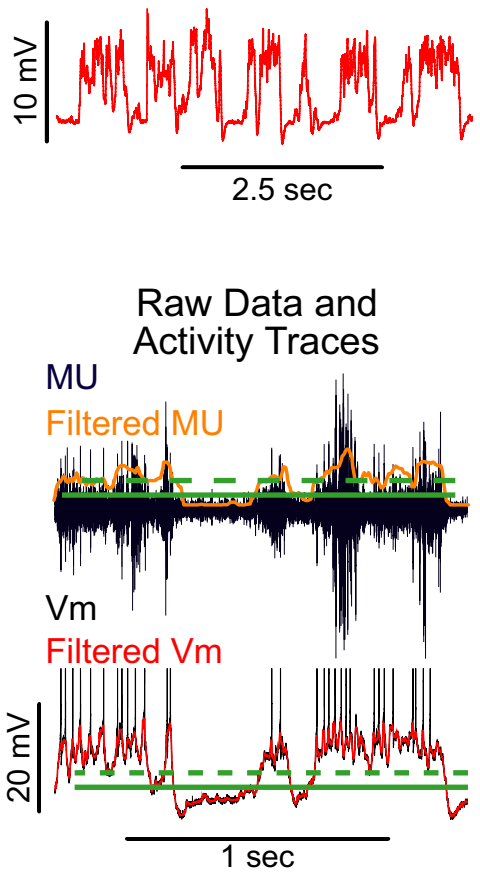

Filtered MU

Overlaid Activity Traces

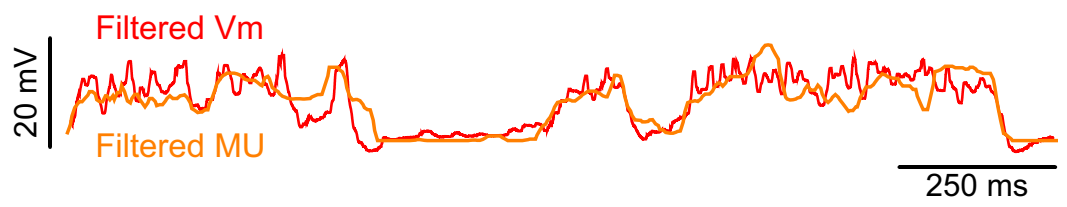

Figure 1. Up states during spontaneous activity in rat barrel cortex. $A$, Multiple-unit (top) and intracellular (bottom) recordings of spontaneous activity from the somatosensory cortex of anesthetized rats. $\boldsymbol{B}$, Activity traces used for identification of Up and Down states. Top, Rectified, median-filtered, and smoothed multiple-unit recording. Bottom, Median-filtered and smoothed intracellular recording. C, Activity histograms constructed from the voltage (red) and MU (orange) traces. Thresholds were placed just above the Down state activity level (solid lines) and at approximately the 50th percentile of the activity distribution (dashed lines). Both multiple-unit activity and membrane potential were clearly bimodal, and plotting the activity measure obtained from the $\mathrm{MU}$ as a function of the activity measure obtained from the intracellular recording revealed a close correspondence between the two traces (black). D, Top, Relationship between the MU (black), the MU-derived activity trace (orange), and the MU-derived upper and lower thresholds (top green solid and dashed lines, respectively) and between the intracellular voltage (bottom, black), the voltage-derived activity trace (bottom, red), and the voltage-derived upper and lower thresholds (bottom green solid and dashed lines). $\boldsymbol{E}$, Expanded MU-based activity trace (orange) and $V_{\mathrm{m}}$-based activity trace (red) showing the close relationship between local multiple-unit activity and intracellular $V_{\mathrm{m}}$.

an increased baseline firing rate (from $0 \mathrm{~Hz}$ during the Down state to $17 \pm 14 \mathrm{~Hz} ; n=16$ cells) (Fig. $3 B$ ). The Up state, compared with the Down state, modestly increased the spike rate after small whisker deflections (Fig. $3 B, C$, solid lines) (calculated over the $50 \mathrm{~ms}$ after stimulus) and significantly decreased the response latency, both to onset of the first spikes and to all spikes occurring in the $50 \mathrm{~ms}$ after stimulus onset (Fig. $3 B-D$ ). However, many of these additional spikes were a consequence of the high baseline spike rate. After subtracting the baseline spike rate (calculated over the $50 \mathrm{~ms}$ before stimulus) from the input-output curves, the Up state clearly reduced neuronal responsiveness to all sizes 
A
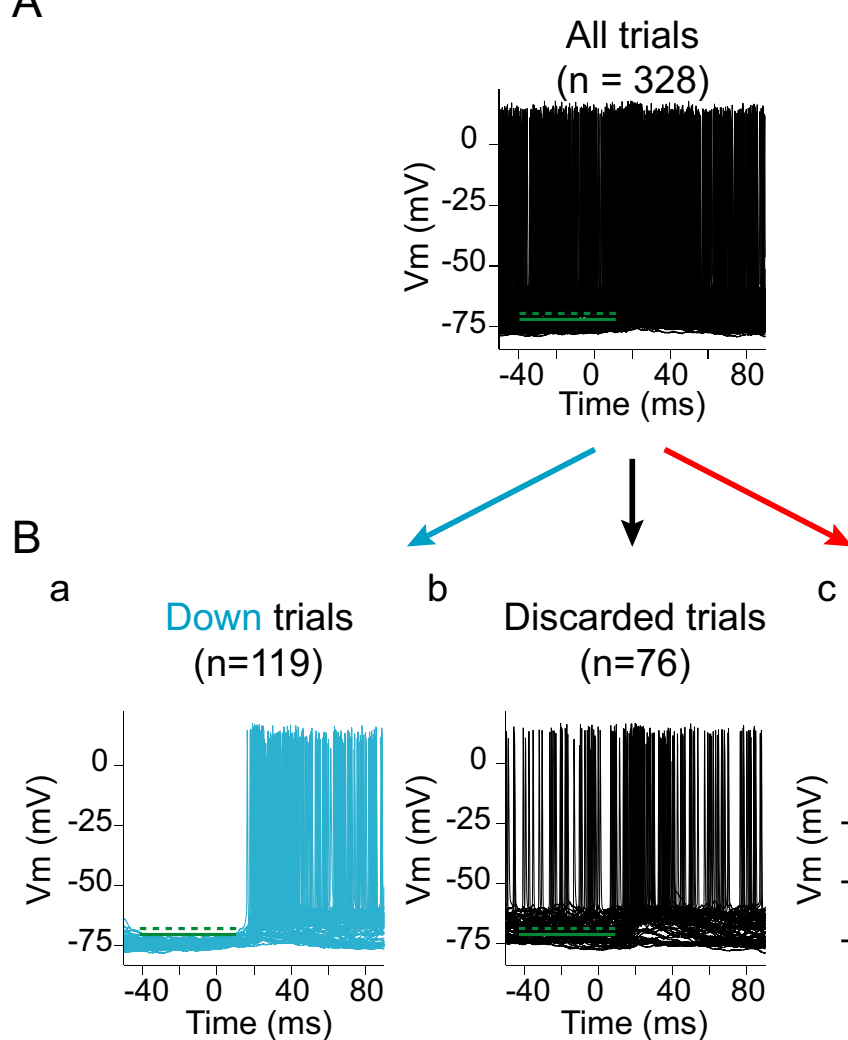

$(n=76)$

Up trials

$(n=133)$
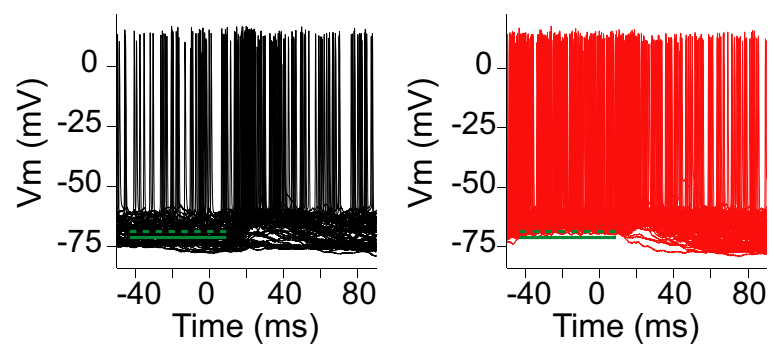

C
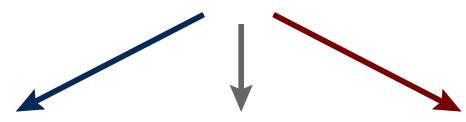

a

Up state started just before trial $\mathrm{b}$
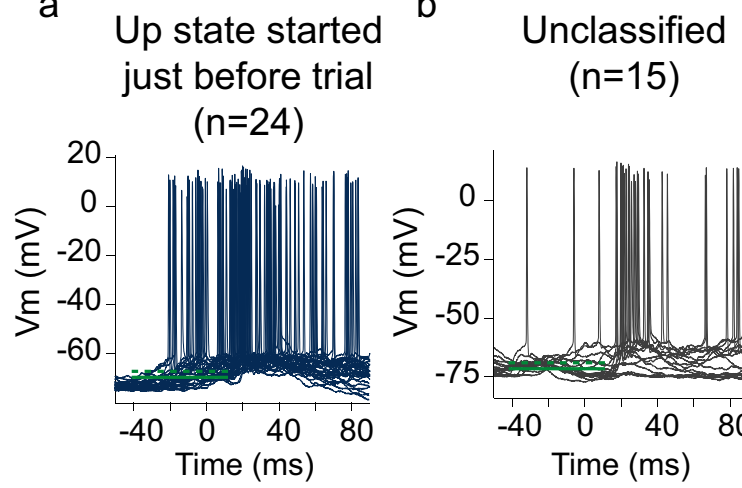

$(n=15)$

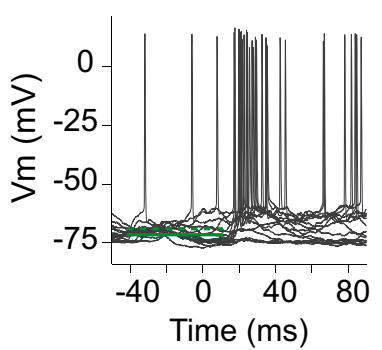

C Up state ended just before trial $(n=37)$

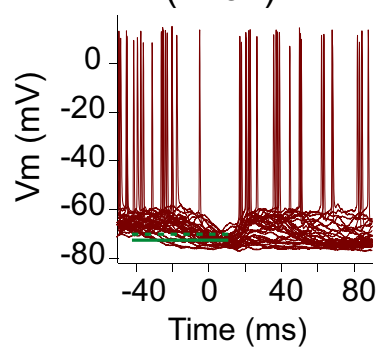

Figure 2. Determination of cortical state before whisker stimulation. $A$, Black, Overlays of the intracellular voltage responses to $n=328$ presentations of a brief whisker stimulus. Green lines, Upper (dotted) and lower (solid) thresholds obtained from the intracellular activity histogram in Figure 1C. The lines extend over the duration for which the pretrial voltage (time shifted by $10 \mathrm{~ms}$ to compensate for system latency) is inspected for classification. $\boldsymbol{B}$, Classification of trials into Up, Down, and Discarded. $\boldsymbol{B a}$, Trials whose voltage, during the $50 \mathrm{~ms}$ pretrial, remained below the lower threshold, were classified as Down trials. $\boldsymbol{B} \boldsymbol{b}$, Trials whose voltage crossed both the upper and lower thresholds were discarded. $B \boldsymbol{C}$, Trials whose voltage passed above the upper threshold at least once and never passed below the lower threshold were classified as Up trials. C, Analysis of discarded trials. $\mathbf{C a}$, In $\sim 40 \%$ of discards, the voltage began below the lower threshold and passed above the upper threshold during the $50 \mathrm{~ms}$ pretrial. $\boldsymbol{C b}$, In $\sim 20 \%$ of discards ( $\sim 5 \%$ of total trials), the pretrial voltage crossed the upper and lower thresholds multiple times. $C c$, In $\sim 40 \%$ of discards, the voltage began above the upper threshold and passed below the lower threshold during the $50 \mathrm{~ms}$ pretrial.

by the Up state was not a consequence of an inability of the neuron to increase its responsiveness (e.g., a ceiling effect), because the Up state clearly enhanced the response to the intracellular injection of large, artificial EPSP conductances (Fig. 4), and because additional depolarization during the Up state increased the spontaneous spike rate during the Up state, the number of spikes generated after a stimulus delivered during the Up state, and the baseline-subtracted spike response (Fig. 5).

\section{Up states in barrel cortex facilitate responsiveness to known conductance stimuli}

These results indicate that the Up state in whisker barrel cortex is associated with a reduction (after subtraction of baseline activity) in neuronal responsiveness to transient whisker stimulation. One explanation for this finding could be that the Up state in rodent primary somatosensory cortex reduces the responsiveness of cortical neurons to their synaptic inputs: for example, if the Up state were associated with strong inhibition or a dramatically decreased membrane resistance in cortical neurons or if the neurons of whisker barrel cortex were intrinsically less responsive during tonic depolarization. An alternative explanation is that the Up state might alter or reduce the whisker-evoked synaptic response. To test whether Up states facilitate or diminish neuronal responsiveness, we examined the responses of cortical neurons, as a function of cortical state, to the intracellular injection of artificial EPSP conductances (Fig. 4A) (Sharp et al., 1993; Dorval et al., 2001; Shu et al., 2003b). The Up state was found to clearly increase the probability that a given excitatory conductance would be followed by an action potential (Fig. $4 B$, solid lines). The Up state also caused an increase in baseline spike rate, from no firing during the Down state to $10-35 \mathrm{~Hz}$ in the Up state. However, even after subtracting the baseline firing rate, the Up state increased the likelihood of action potential generation after all but perhaps the largest conductance stimuli (Fig. 4B, dotted line). Across $n=9$ neurons, the Up state increased the output spike rate in response to all sizes of artificial PSP (Fig. 4C). of whisker deflections compared with the Down state (Fig. 3C, dotted line). This reduction was consistent from cell to cell (Fig. $3 D$ ) (all spike counts normalized to maximum; maximum response, $40 \pm 22 \mathrm{~Hz}$ in the Down state; $38 \pm 20 \mathrm{~Hz}$ in the Up state; $24 \pm 20 \mathrm{~Hz}$ in the Up state with baseline subtraction). The lack of enhancement of the response to the largest whisker movements

\section{DC-induced depolarization increases responsiveness to} whisker stimuli

It has been proposed (Sachdev et al., 2004) that the tonic depolarization of the Up state reduces neuronal responsiveness by, among other things, increasing action potential threshold, reducing the driving force on excitatory conductances, and increasing 
the driving force on inhibitory conductances. Measurements of the voltage threshold during Up and Down states showed that the voltage threshold for the first spike after a stimulus was increased only modestly by $1.8 \pm 1.1 \mathrm{mV}$ during the Up state $(n=11$ cells; $p<0.001$; data not shown). However, to strictly control for changes in responsiveness attributable solely to changes in membrane potential, we compared the responsiveness of cortical neurons to stimuli delivered in the Up and Down states when the cell was at its natural Down state membrane potential (Fig. 5Aa) and when the cell was depolarized so that the membrane potential of the Down state equaled the natural membrane potential of the Up state (Fig. $5 A b$ ). In the Down state, the whisker-evoked synaptic conductance delivered to the cortical neuron should be essentially identical whether or not the neuron is depolarized, allowing us to directly test the effect of membrane potential on neuronal responsiveness.

We found that, whereas the spontaneous depolarization of the Up state was associated with a strong decrease in responsiveness to all stimuli tested (compared with the unmodified Down state), experimenter-induced depolarization such that the Down state membrane potential matched that of the normal Up states resulted in a strong increase in responsiveness to vibrissal stimuli (compared with the unmodified Down state) (Fig. 5B). Overall, each millivolt of spontaneous depolarization during the Up state was associated with a decrease in responsiveness of $0.6-1.6 \mathrm{~Hz}$ (baselinesubtracted change in response to medium amplitude stimulus, $-0.6 \pm 0.5 \mathrm{~Hz}$; large stimulus, $-1.6 \pm 1.0 \mathrm{~Hz} ; n=5$ cells). However, each millivolt of experimenterinduced depolarization during the Down state was associated with an increase in responsiveness of 1-2 $\mathrm{Hz}$ (baselinesubtracted change in response to medium amplitude stimulus, $1.0 \pm 1.9 \mathrm{~Hz}$; large stimulus, $2.0 \pm 1.3 \mathrm{~Hz} ; n=5$ cells) (Fig. $5 C)$. In addition, experimenter-induced depolarization during the Up state was associated with a modest increase in responsiveness, even after baseline subtraction, indicating that neuronal responsiveness was not saturated during the Up state (change in responsiveness to large stimulus, $1.4 \pm 1.2 \mathrm{~Hz}$ before baseline subtraction and $0.7 \pm 0.5 \mathrm{~Hz}$ after baseline subtraction; $n=5$ cells) (Fig. $5 A, B$ ). One caveat of these experiments is that natural depolarizations occur through the bombardment of the cell throughout its dendritic tree by synaptic potentials, whereas depolarization induced by the intracellular injection of current is centered around the tip of the recording electrode, which is typically located in or near the soma. However, because action po-

C

a
A

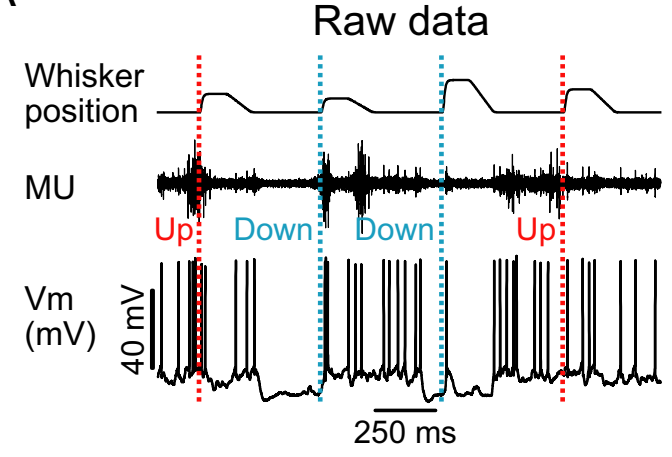

B

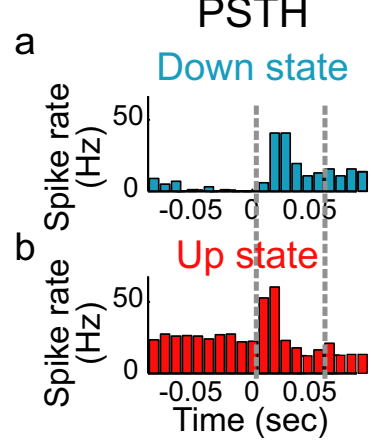

Responsiveness and latency
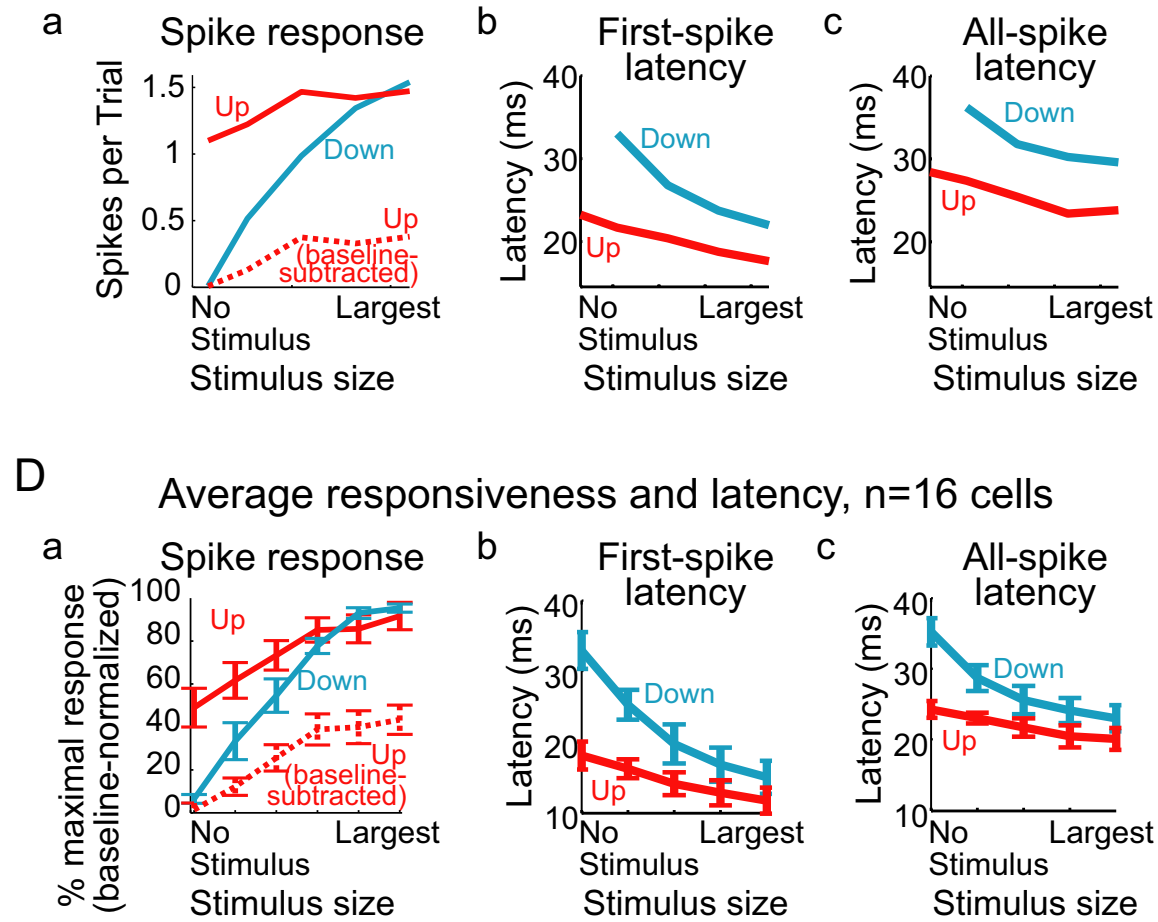

Figure 3. Up states decrease responsiveness to brief whisker deflections. $\boldsymbol{A}$, While recording intracellularly from a cortical neuron, the principal whisker for that neuron was stimulated with random-amplitude ramp-and-hold deflections (stimulus times marked with dotted lines). In response to both a weak and a strong stimulus delivered during the Down state, the cortical neuron responded with a large PSP and clear increase in spike rate; however, the neuron responded with no obvious PSP and no obvious increase in spike rate to the stimulus delivered during the Up state. $\boldsymbol{B}$, The PSTHs for one stimulus size, sorted according to whether the stimulus occurred during the Down state (top) or the Up state (bottom), show that stimuli delivered during the Down state elicited a clear increase in spike rate, whereas stimuli delivered during the Up state caused a more modest increase in spike rate relative to baseline. Dashed lines delineate times over which spikes were counted for the input- output curves in C. C, Poststimulus spike counts for a range of stimulus sizes show that, although weak stimuli are followed by more spikes if they are delivered during the Up state (red), most of these spikes are associated with the baseline firing of the cell. Baseline subtraction of Up state responses (red, dashed) reveals a reduction in responsiveness to all stimulus sizes relative to the Down state (blue). $\boldsymbol{D}$, Normalized input- output curves for $n=11$ cells show that, for weak stimuli, the Up state (red, solid) increases the number of spikes generated after a stimulus compared with the Down state (blue). Baseline subtraction reveals reduced responsiveness during the Up state to all stimulus sizes (red, dashed). Error bars represent SE.

tentials are initiated in the axon initial segment (Shu et al., 2007), all but axo-axonic synaptic inputs must propagate through the soma to reach the point of spike initiation. Thus, manipulations of the somatic membrane potential (or the intrasomatic injection of artificial synaptic conductances) with current injection may mimic to a large extent the effects on action potential generation of spontaneous changes in membrane potential occurring with natural synaptic activity (Shu et al., 2003b). We conclude that 
A

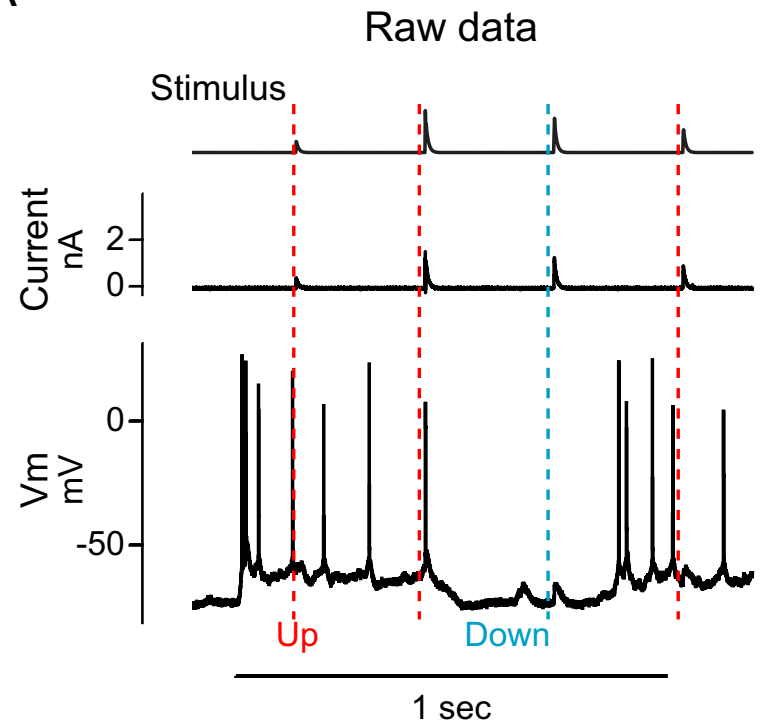

B

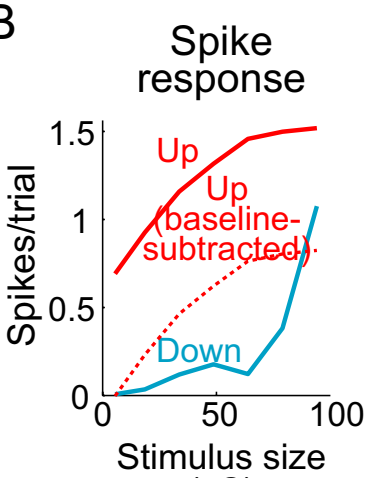

(nS)
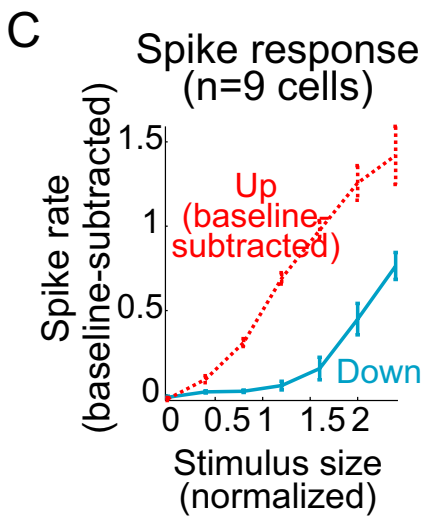

Figure 4. Up states increase responsiveness to synthetic EPSPs. $\boldsymbol{A}$, Random-amplitude AMPA-like excitatory conductances were injected into the cortical neuron during Up states (trials marked with red lines) and Down states (blue line). B, Input- output curves were constructed for Up and Down state trials separately; all stimuli were followed by more spikes if delivered during an Up state (red, solid) compared with a Down state (blue). After baseline subtraction (red, dotted), Up states remained facilitatory to all but the largest stimuli in this cell. C, Averaged input- output curves in $n=9$ neurons show facilitation of spike responsiveness to all stimulus sizes by the Up state compared with the Down state, even after baseline subtraction. Error bars represent SE.

depolarization-induced decreases in neuronal excitability (such as might result from inactivation of voltage-gated currents or changes in driving force on PSPs) are unlikely to account for the decrease in responsiveness to whisker stimuli during Up states.

\section{Whisker stimuli evoke state transitions}

One possible explanation for the difference in neuronal responsiveness to stimulus-evoked PSPs, which is decreased by the Up state, and to the injection of artificial EPSPs, which is enhanced by the Up state, is that whisker stimulation may actually be causing cortical networks to transition between Up and Down states. For example, if whisker stimulation during the Down state resulted in a transition into the Up state, this network transition may initiate action potentials that would be counted as part of the sensory response. Naturally, this would not be the case with the intracellular injection of artificial EPSPs. Indeed, previously, we demonstrated that the activation of cortical inputs by white matter electrical stimulation could both initiate and terminate Up

A

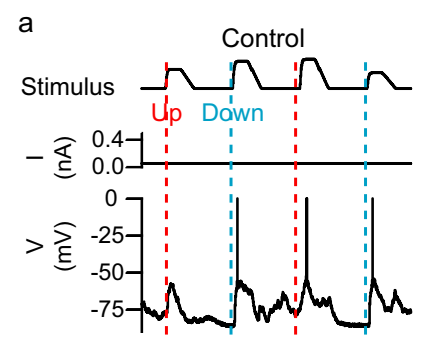

b

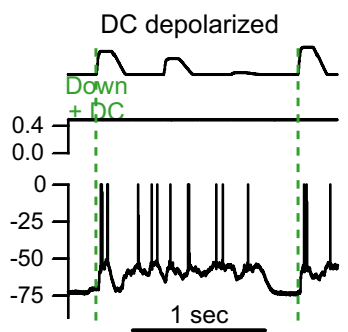

B

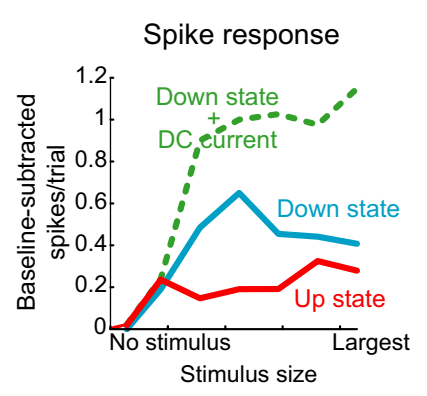

C

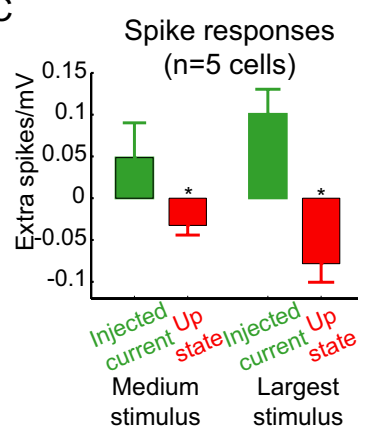

Figure 5. DC depolarization and network-induced depolarization have opposite effects on responsiveness to whisker stimulation. $A$, Random-amplitude ramp-and-hold whisker stimuli were delivered during Up states (trials marked with red lines) and Down states (trials marked with blue lines). $\boldsymbol{B}, \mathrm{DC}$ was injected to depolarize the Down state to the level of the Up state, and random-amplitude whisker stimuli were delivered every $500 \mathrm{~ms}$. Only Down state trials were examined (green lines). C, Baseline-subtracted input- output curves were constructed for Up states (red), Down states (blue), and depolarized Down states (green). At a variety of stimulus sizes, Up states diminished neuronal responsiveness, whereas current-induced depolarization that caused depolarization similar in amplitude to real Up states increased neuronal responsiveness. $\boldsymbol{D}$, Across $n=5$ cells, experimenter-induced (DC) depolarization increased neuronal responsiveness to both strong and weak stimuli, whereas spontaneous (Up state-induced) depolarization diminished neuronal responsiveness to both strong and weak stimuli. Error bars represent SE. ${ }^{*} p<0.05$.

states (Shu et al., 2003a). We therefore examined whether the whisker deflection could evoke transitions to the Up state and whether these transitions could explain the observed changes in responses. If the whisker deflection was delivered during a Down state, it appeared to the naked eye to be often followed within 50 $\mathrm{ms}$ by the initiation of an Up state (Figs. $2 \mathrm{Ba}, 6$ ). Likewise, if the whisker deflection was delivered during an Up state, it often appeared to be followed by the termination of the Up state (Figs. $2 B c, 6)$. We quantified this tendency by measuring the duration of stimulus-evoked depolarizations (Fig. 6B,Da,Ea). The distributions of depolarization durations after stimuli delivered during either the Up or Down states were significantly bimodal (Down, $p=0.04 ; \mathrm{Up}, p=0.01$; Hartigan's dip test; significant at $p \leq 0.05$ for Down state duration distributions in 9 of 12 cells and for Up state duration distributions in 12 of 12 cells). These results suggest that a significant number of whisker stimuli are followed by transitions between cortical states.

To investigate this further, we plotted the probability of state transition as a function of time from whisker stimulation (Fig. $6 C)$. Whisker stimulation resulted in both a significant increase in the probability of Down-to-Up state transitions (Fig. 6Ca) ( $p<0.01$ for the $50 \mathrm{~ms}$ after a whisker stimulus; $\chi^{2}$ test; dots indicate state transitions, not action potentials) and Up-to-Down transitions (Fig. 6Cb) $(p<0.05)$. Down-to-Up transitions were significantly enhanced by whisker stimulation in 12 of 12 record- 
A

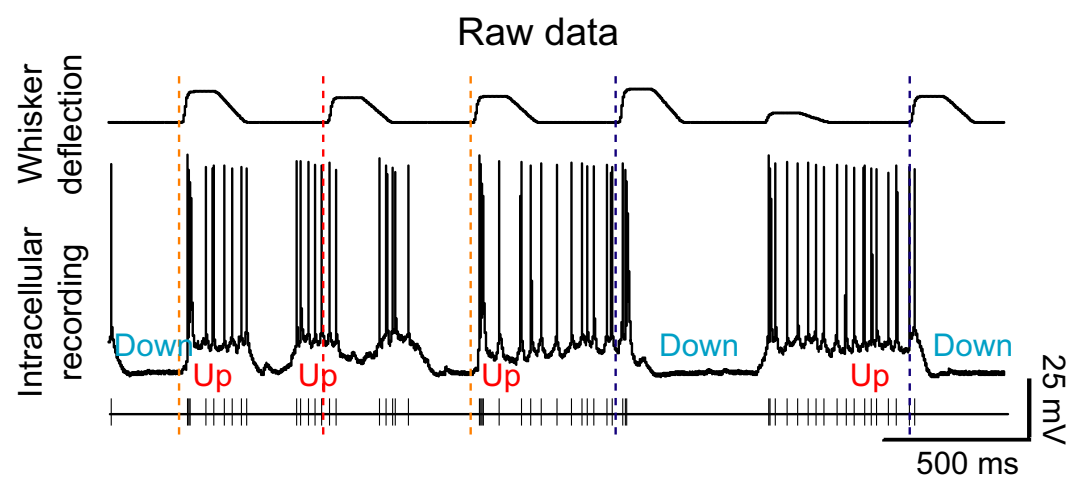

B
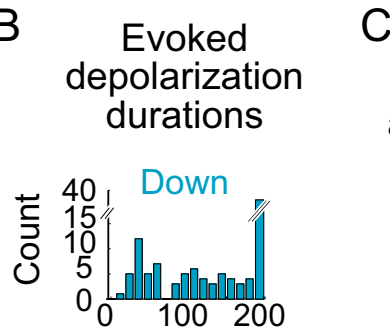

a

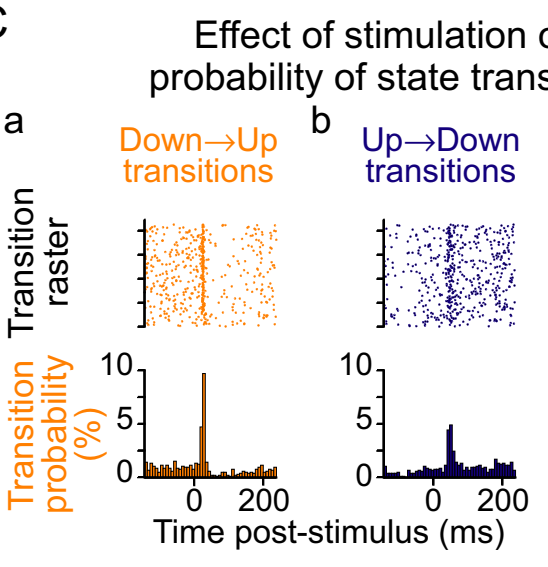

ition

C Group data $\mathrm{n}=12$ cells

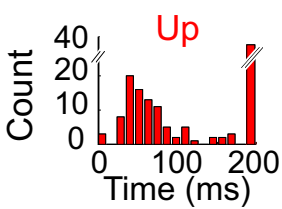

$\mathrm{Da}$

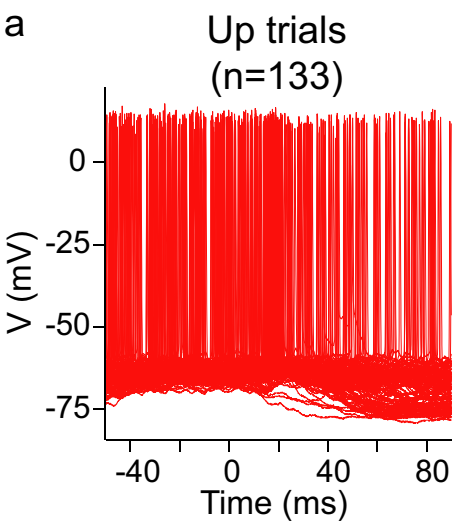

b

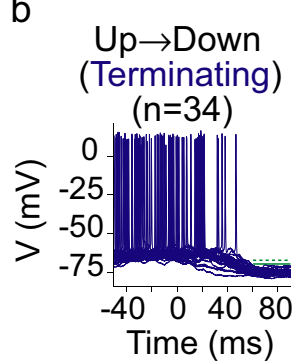

c

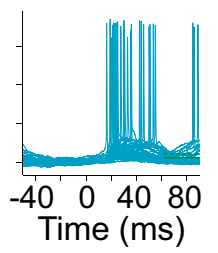

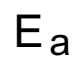

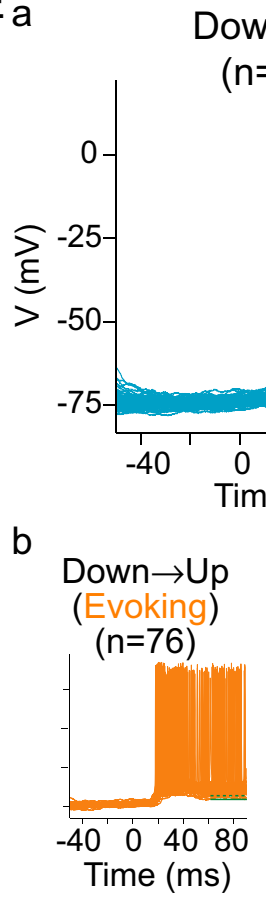

ings, and Up-to-Down transitions were significantly enhanced in $n=4$ of 12 recordings (Fig. $6 C c$ ).

These data suggest that the apparent ability of Down states to facilitate responsiveness to whisker stimuli might in fact be mediated by the explosive evocation of recurrent activity by whisker stimuli. We therefore further classified stimuli delivered during the Up state (Fig. 6Da) according to whether the membrane potential trajectory returned to the Down state membrane potential ("Up-to-Down" or "Terminating" trials) (Fig. $6 D b$ ) or stayed within the range of Up state membrane potentials ("Up-to-Up" trials) (Fig. 6Dc) immediately after the stimulus-evoked PSP (see Materials and Methods). Similarly, we further classified stimuli delivered during the Down state (Fig. 6 Ea) according to whether the membrane potential remained within the distribution of Up state membrane potentials ("Down-to-Up" or "Evoking" trials) (Fig. 6 Eb) or returned to baseline ("Down-to-Down" trials) (Fig. $6 E c)$ immediately after the stimulusevoked PSP.

PSTHs of spike responses to stimuli in each of these four categories were constructed, and the stimulus-evoked change in spike rate was measured (Fig. 7A). For the largest stimulus size tested, Down-toDown stimuli increased neuronal firing rate by $25 \pm 19 \mathrm{~Hz}$. Stimuli that evoked Down-to-Up transitions increased firing rate by $40 \pm 24 \mathrm{~Hz}$, a mean increase of $83 \%$ over the spike rate after Down-to-Down trials. Stimuli presented on Up-to-Up trials were found to increase neuronal firing rate by $17 \pm 15 \mathrm{~Hz}$, a mean decrease of 20\% compared with Down-to-Down stimuli. Finally, stimuli evoking Up-toDown transitions did not significantly alter neuronal firing: firing rate changed by $2 \pm 14 \mathrm{~Hz}$ relative to baseline, a decrease of $94 \%$ compared with Down-to-Down trials $(n=12$ cells; $p<0.01)$.

Notably, when poststimulus spike rates were computed without baseline subtrac-

Figure 6. Brief whisker stimulation evokes cortical state transitions. $A$, Whisker stimulation (times marked with dashed lines) was often observed to be closely followed by state transitions in the intracellular membrane potential. $\boldsymbol{B}$, Stimulus-evoked PSP durations (defined as the time from whisker stimulation to the next crossing of the lower threshold) were significantly bimodal for both Down- (blue) and Up- (red) delivered stimuli (Hartigan's dip test, Down, $p=0.037$; Up, 0.012 ; significantly bimodal at $p \leq$ 0.05 for 9 of 12 Down PSP duration distributions and 12 of 12 Up PSP duration distributions). Ca, Stimuli delivered during the Down state were followed, in the next $50 \mathrm{~ms}$, by a higher-than-baseline rate of Down-to-Up state transitions. [Note that dots represent Down-to-Up transitions (see Materials and Methods) and not action potentials.] $\boldsymbol{C} \boldsymbol{b}$, Stimuli delivered during the Up state were also, in the next $50 \mathrm{~ms}$, followed by a higher-than-baseline rate of Up-to-Down state transitions (same cell as $\boldsymbol{A}$; dots represent Up-to-Down transitions). Cc, Across $n=12$ cells, the rate of Down-to-Up state transitions was increased by a factor of 4 in the 50 $\leftarrow$

ms after whisker stimulation (significant in 12 of 12 cells individually), whereas the rate of Up-to-Down state transitions was increased by a factor of 1.5 in the 50 ms after whisker stimulation (significant in 5 of 12 cells individually). D, Trials previously classified as Up trials $(\boldsymbol{a})$ were therefore subclassified according to whether the stimulus was $(\boldsymbol{b})$ or was not (c) followed, in the next $50-80 \mathrm{~ms}$, by a crossing of the lower voltage threshold. $\boldsymbol{E}$, Trials previously classified as Down trials (a) could be subclassified according to whether the poststimulus voltage remained above the lower threshold $(\boldsymbol{b})$ or fell below the lower threshold (c) at $50 \mathrm{~ms}$ after trial. Error bars represent SE. ${ }^{*} p<0.05$. 
A

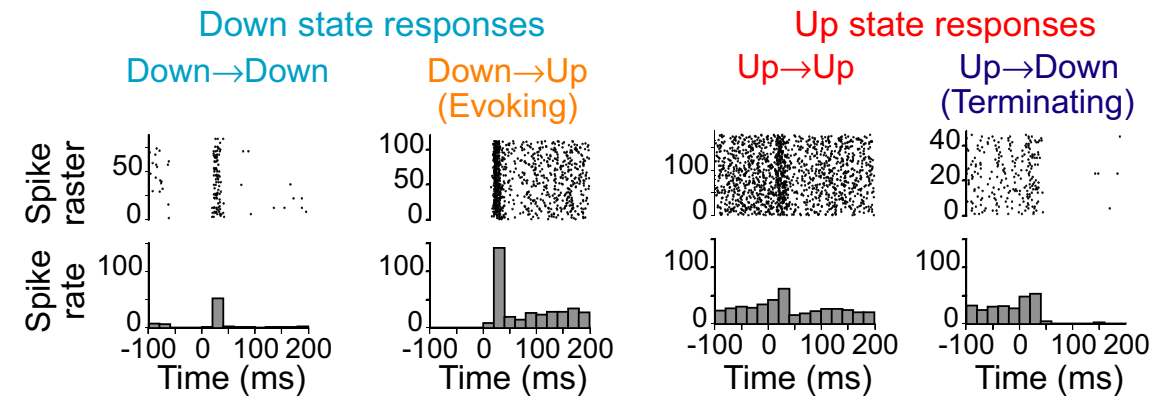

State dependence of spike responses neuronal responsiveness was significantly less than responsiveness $300-400 \mathrm{~ms}$ into the Down state $(n=12$ cells; $p<0.05)$. Similarly, during the first $100 \mathrm{~ms}$ of the Down state, neuronal responsiveness was significantly greater than neuronal responsiveness between 300 and $400 \mathrm{~ms}$ into the Up state $(n=12$ cells; $p<0.05)$. In addition, we found that transitions were far less likely to be evoked immediately after a state change. Whisker stimuli presented immediately after the beginning of a Down state almost never evoked transitions to the Up state, but instead evoked 50- to 100-ms-long PSPs (supplemental Fig. 1, available at www.jneurosci.org as supplemental material), whereas whisker stimuli presented $300-400 \mathrm{~ms}$ after the beginning of a Down state almost always evoked Up state transitions (Fig. 7Cc). Notably, the effect of whisker stimulation in evoking transitions was not only related to stimulus timing, but also to stimulus strength: stronger stimuli were more likely to evoke Up state transitions (data not shown).

Together, these results indicate that a significant portion of the increased responsiveness of cortical neurons during the Down state is associated with the ability of stimuli to evoke transitions to the Up state. However, even stimuli delivered during the Down state that do not overtly initiate transitions to the Up state can cause a larger change in spike rate (especially if presented late in the Down state) than do the same stimuli delivered during the Up state.

\section{Network state changes the conductance and reversal potential of stimulus-evoked PSPs}

Figure 7. The ability of whisker stimulation to induce state changes is cyclical and related to sensory responsiveness. A, PSTH for whisker stimulation (same cell as in Fig. 6), separated according to whether the whisker stimulus succeeded or failed in evoking state transition. Note the increased spike generation after a stimulus that successfully evokes an Up state and reduced spike generation after a stimulus that successfully terminates an Up state. $\boldsymbol{B}$, Across $n=12$ cells, sensory responsiveness is greatest for stimuli that evoke an Up state, lesser for stimuli delivered during an Up state, and smallest for stimuli that terminate an Up state. Ca, During the Down state, in $n=12$ cells, average responsiveness (top) increases as a function of time since the termination of the Up state, whereas average membrane potential (bottom) is stable during the course of the Down state. $\boldsymbol{C} b$, During the Up state, in $n=12$ cells, both raw and baseline-subtracted responsiveness (top) decrease over time, whereas mean membrane potential (bottom) is stable. (c, As time since the termination of the Up state increases, the likelihood of whisker stimulation evoking an Up state dramatically increases. Error bars represent SE. ${ }^{*} p<0.05$.

tion, Up states were no longer associated with decreases in neuronal responsiveness: the raw poststimulus spike rate for Upto-Up trials was $33.7 \pm 22.7 \mathrm{~Hz}$, which was not significantly different from the spike rate observed on Evoking trials $(p=$ 0.09). Both Up-to-Up and Evoking trials elicited significantly greater spike rates than Down-to-Down trials $(p=0.01$ and 0.002 , respectively) or Terminating trials $(21.0 \pm 17.9 \mathrm{~Hz} ; p<$ $0.0005)$, whereas Terminating and Down trials did not evoke significantly different spike rates ( $p>0.01$; data not shown).

Both the spike output after whisker stimulation and the ability of a whisker deflection to evoke a state transition depended critically on the history of local circuit activity. During the Down state, spike responses to whisker stimulation in $n=12$ neurons were smallest immediately after the beginning of a Down state (Fig. 7Ca, top), but recovered over time, although over this time period the membrane potential was stable (Fig. 7Ca, bottom). During the Up state, spike responses to whisker stimulation declined as time spent in the Up state increased (Fig. 7Cb, top), whereas membrane potential was stable over time (Fig. $7 \mathrm{Cb}$, bottom). Although whisker responsiveness increased over time spent in the Down state, during the first $100 \mathrm{~ms}$ of the Up state
Our findings indicate that the differences in Down and Up state spike responses are not attributable merely to the effects of the Up state on somatic membrane potential, implying that the stimulus-evoked PSPs themselves may be significantly different between these states. We therefore directly measured the stimulus-associated synaptic conductances (as seen at the soma) after Down state, Up state, and Down-to-Up transition-evoking whisker stimuli. (Stimulus-evoked Up-to-Down transitions were relatively infrequent, and the associated PSPs were too small to reliably measure.) We examined the postsynaptic currents after whisker deflections in $n=6$ neurons recorded with sharp electrodes containing $50 \mathrm{~mm}$ QX-314 (to block active sodium currents, the h-current, and $\mathrm{GABA}_{\mathrm{B}}$ receptor-mediated IPSPs) (Yeh, 1978; Cahalan and Almers, 1979; Stafstrom et al., 1985; Nathan et al., 1990; Perkins and Wong, 1995) and $2 \mathrm{M}$ cesium acetate (to reduce $\mathrm{K}^{+}$currents) at a variety of membrane potentials (Fig. $8 A a, A c, A e)$. This permitted us to estimate the magnitude and reversal potential of the additional conductance associated with a whisker stimulus, by comparing the current-voltage relationship at each time after the stimulus to the current-voltage relationship before stimulus delivery (Fig. $8 A b, A d, A f$ ) (see Materials and 
Methods). In addition, this permitted us to measure the effect of the Up state on neuronal input resistance, by comparing the slopes of the $I-V$ relationships obtained during the Up state to those obtained during the Down state. Although this method does not provide a complete analysis of excitatory and inhibitory inputs to cortical neurons, it is superior to previously used methods (Sachdev et al., 2004) because of the decrease in basal membrane conductance induced by including $\mathrm{Cs}^{+}$in the micropipette and the use of 10-30 trials obtained at a minimum of five different membrane potentials to construct a full $I-V$ plot before and during the whiskerevoked response.

Using this method, we found that the Up state is associated with an increase in membrane conductance of $22 \pm 13 \mathrm{nS}$ $(100 \pm 60 \%)$, as previously reported for Up states in the ferret prefrontal cortex (Shu et al., 2003a; Haider et al., 2006). In addition, the peak magnitude of the stimulus-evoked change in membrane conductance during Down-to-Down trials was $23 \pm 10 \mathrm{nS}$. On Down-to-Up stateevoking trials, the same stimuli were followed by much larger PSPs, possessing an average peak increase in conductance of $54 \pm 28 \mathrm{nS}$. On these Down-to-Upevoking trials, stimuli caused peak conductance changes that varied from 66 to $640 \%(168 \pm 188 \% ; p<0.05)$ greater than the peak conductance changes during Down-to-Down trials. In contrast, stimuli presented on Up-to-Up trials did not evoke a substantially larger peak conductance change than on Down-to-Down trials. The peak conductance change during an Up-to-Up trial was $26 \pm 15 \mathrm{nS}$, a nonsignificant increase of $20 \pm 60 \%$ over that observed on Down-to-Down trials. Unfortunately, the combination of increased conductance and increased membrane noise during the Up state, compared with the Down state, limited our ability to determine the timing of PSP onset or offset and thus to compare the durations of PSPs evoked from the Up and Down states. However, the PSP peak occurred earlier on Up trials than Evoking trials (by an average of $14 \pm 10 \mathrm{~ms}$ ) or Down trials (by an average of $4 \pm 3 \mathrm{~ms}$ ).

Although the overall magnitudes of the conductances evoked by whisker stimulation were similar during Up and Down states, the reversal potentials (as measured at or near the peak response) were significantly different. Stimulus-evoked PSPs during the Up state reversed at an average of $-56.3 \pm 3.1 \mathrm{mV}$, which is $3-15 \mathrm{mV}$ more negative than either of the Down state stimulus-evoked PSPs. The Down state trials that evoked Up states possessed PSPs that re-

A represent SE.
Down

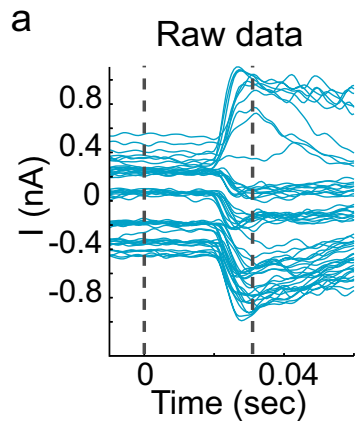

b

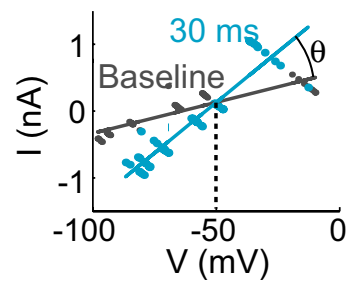

B

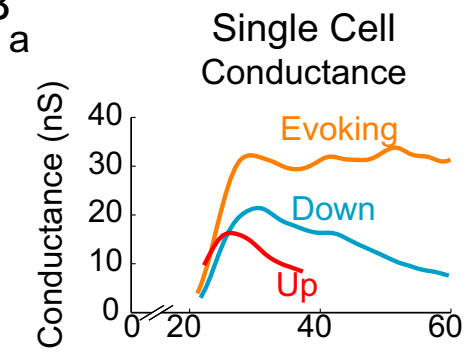

b

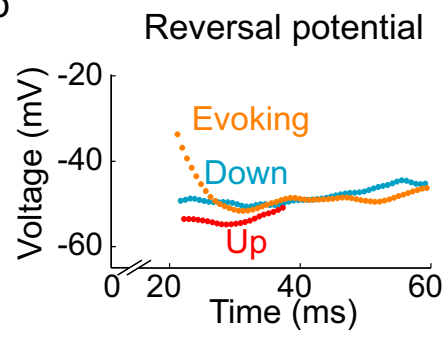

Evoking

C

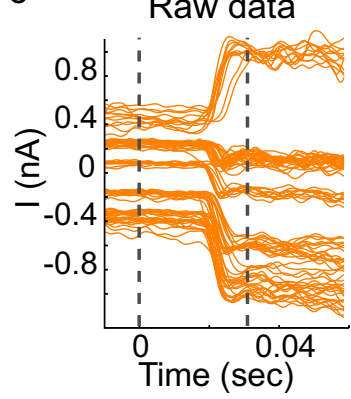

d

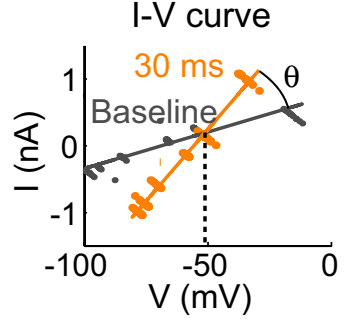

Up

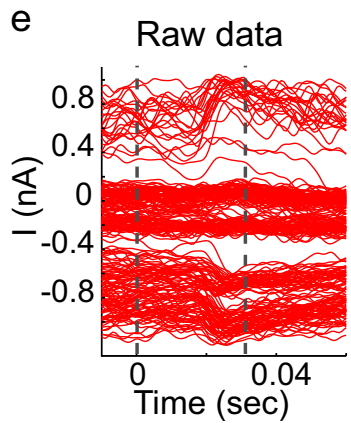

f

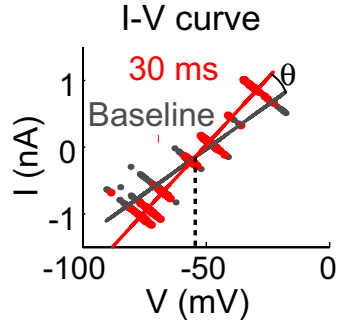

C

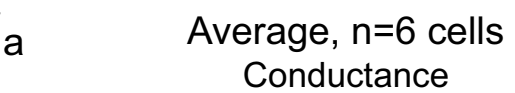

Figure 8. Whisker deflection evokes a smaller conductance change during the Up state. $\boldsymbol{A}$, Voltage-clamp recordings of the stimulus-evoked PSC after Down state stimuli that failed $(\boldsymbol{a}, \boldsymbol{b})$ and succeeded $(\boldsymbol{c}, \boldsymbol{d})$ in evoking Up states, as well as of Up state stimuli $(\boldsymbol{e}, \boldsymbol{f})$, at a variety of membrane potentials. $\boldsymbol{A} \boldsymbol{b}, \boldsymbol{A d}, \boldsymbol{A f}, I-V$ plots were constructed; the point of intersection of the $I-V$ curves was taken to be the reversal potential of the stimulus-evoked conductance, and the difference in slopes $(\theta)$ between the I-V curves was taken to be the magnitude of the stimulus-evoked conductance. $B \boldsymbol{a}$, Although the magnitude of the stimulusevoked conductance was initially similar for Up, Down, and Evoking trials, the conductance size developed a larger peak magnitude, and remained larger, for trials that succeeded in evoking Up states than for trials delivered during the Up state or for Down state trials that failed to evoke an Up state. $\boldsymbol{B} \boldsymbol{b}$, The reversal potential of the stimulus-evoked conductance was slightly more negative for Up state stimuli than for stimuli delivered in the Down state, regardless of whether they evoked an Up state. Ca, Across $n=6$ cells, the magnitude of the evoked conductance was significantly larger for Evoking stimuli than for Down or Up stimuli, whereas the total evoked conductance magnitude was not significantly different between Down or Up stimuli. $\boldsymbol{C} \boldsymbol{b}$, The reversal potential of the evoked conductance was significantly more negative for Up stimuli than for Evoking or Down stimuli and was not significantly different between stimuli delivered during the Down state that succeeded or failed in evoking Up states. Error bars versed at $-47.4 \pm 3.8 \mathrm{mV}$, whereas the non-evoking trials had PSPs with a reversal potential of $-43.2 \pm 5.7 \mathrm{mV}(p<0.05)$ (Fig. $8 \mathrm{Cb}$ ). In contrast, comparison of the reversal potential during Down-to-Down- and Down-to-Up-evoking trials did not reveal 
A

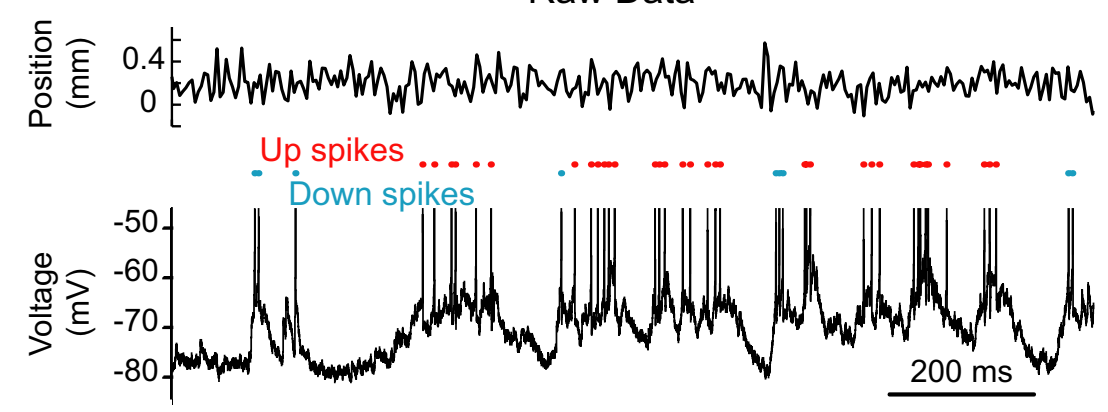

B

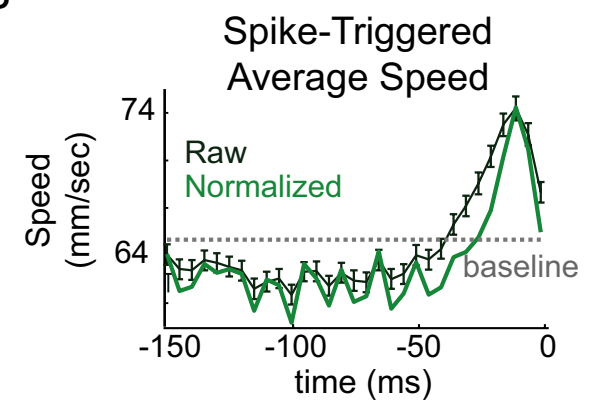

D

\section{Scales and correlations of pre-spike stimuli}

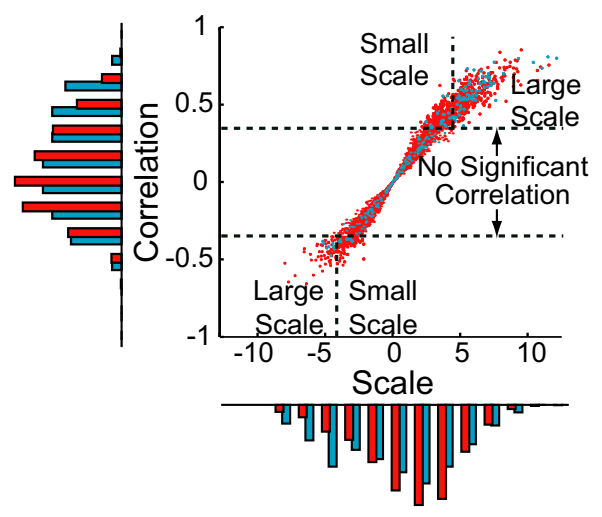

C

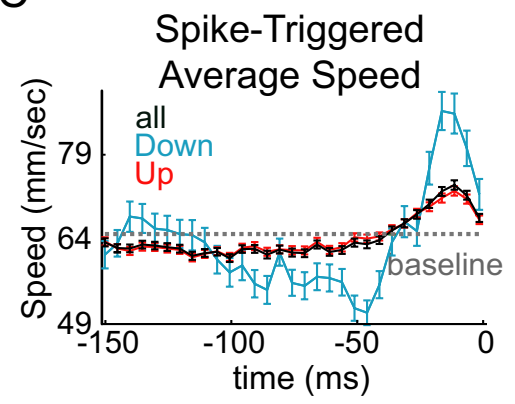

$E$

\section{$E$}

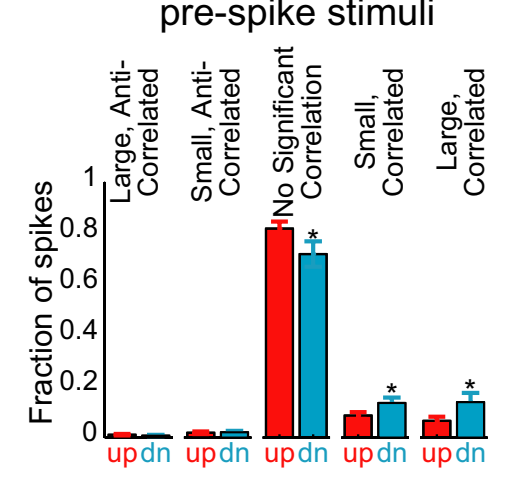

Figure 9. Network activity substantially increases spike rate while decreasing spike precision. $\boldsymbol{A}$, During continuous random whisker stimulation (top), almost all action potentials were generated from the Up state (bottom, red dots) rather than from the Down state (bottom, blue dots). $\boldsymbol{B}$, Spike-triggered average of the whisker speed shows the neuron's average prespike speed (black) and autocorrelation-corrected prespike speed (the kernel or STRF; green): this neuron exhibited the characteristic pattern of reduced speed followed by increased speed before action potential generation. C, Spike-triggered averages of whisker speed for Up and Down state spikes separately show that the spike-triggered averages of speed for Up and Down states have the same shape, but the Down state STRFs are more strongly modulated. D, Plot of the correlation and scale of each prespike stimulus, compared with the STRF, separated into spikes generated from Up and Down states. The scale factor ( $x$-axis and bottom histogram) is the amount by which the average prespike stimulus (STRF) would have to be stretched to best fit (see Materials and Methods) the actual prespike stimulus: a large positive value means that the STRF had to be greatly stretched to minimize the difference between this and the actual prespike stimulus, and a large negative value means that the STRF had to be stretched and inverted to minimize the difference between the STRF and the actual prespike stimulus. The correlation ( $y$-axis and left histogram) is the Pearson's correlation coefficient between the prespike stimulus and the STRF. The correlations were separated into those that were or were not statistically significant at the $p=0.05$ level. $E$, Across $n=5$ cells, no significant differences were found in the fraction of Up state and Down (dn) state spikes preceded by stimuli that were significantly anticorrelated with the STRF. A significantly greater fraction of Up state spikes were preceded by stimuli uncorrelated with the STRF. A significantly greater fraction of Down state spikes were preceded by stimuli that were significantly correlated with the STRF, both large and small; however, among significantly correlated stimuli, a greater proportion were small-scale among Up state spikes than among Down state spikes. Error bars represent SE. ${ }^{*} p<0.05$.

a significant difference (Fig. $8 \mathrm{Cb}$ ). The reduction in neuronal responsiveness during Up states is therefore associated with two changes in the characteristics of the stimulus-evoked conductances. First, the conductances after stimuli delivered during Up states exhibit a more negative reversal potential (indicating a greater proportion of inhibition vs excitation) than those delivered during Down states. Second, PSPs after stimuli that successfully evoke Up states, which typically fall later in the Down state than PSPs that fail to evoke Up states, exhibit a larger change in conductance than either PSPs after stimuli delivered during the Up state or PSPs after Down-delivered stimuli that fail to evoke an Up state.

\section{Effect of Up and Down states on neuronal responsiveness to prolonged stimuli}

We have seen that neuronal responsiveness to whisker stimulation is the product of an interaction between cortical state and stimulus characteristics and that brief and infrequent whisker stimuli evoke stronger increases in firing rate over baseline in the Down state than in the Up state. However, during normal behavior, whiskers may contact surfaces continuously and/or irregularly for tens or hundreds of milliseconds at a time, causing the whiskers to vibrate in a complex manner (Arabzadeh et al., 2005; Hipp et al., 2006). We therefore used continuous, fluctuating whisker movements to examine the effects of Up and Down states on neuronal responsiveness to continuous whisker stimulation. Specifically, we addressed two questions. First, how does cortical state affect the overall level of neuronal responsiveness to prolonged stimulation? And second, how does cortical feature selectivity depend on cortical state?

\section{Effect of Up and Down states on \\ feature selectivity}

When continuous, fluctuating whisker movements (see Materials and Methods) were delivered, the majority of the action potentials observed in cortical neurons ( $n=6$ cells) were either preceded by or followed by periods of persistent depolarization. In other words, almost all of the spikes generated during continuous whisker stimulation were generated during Up states (80-98\%; mean, $85 \pm 10 \%, n=6$ cells), and a clear relationship between the stimulation of the whisker and the membrane potential fluctuations and spike generation in the cortical neuron was not immediately evident (Fig. 9A). To estimate 
the feature selectivity of the neurons, autocorrelation-corrected spike-triggered averages of the whisker position, velocity, and speed were constructed (where speed is rectified velocity). Despite the lack of an obvious relationship between stimulus and spikes, clear receptive fields were obtained for all neurons: although five of the six neurons did not exhibit a clear receptive field with respect to whisker position or velocity, all six neurons showed clear receptive fields consisting of a prolonged (50-500 $\mathrm{ms})$ reduction in speed (compared with the mean whisker speed) followed by a transient $(10-50 \mathrm{~ms})$ increase in speed (Fig. 9B). To compare feature selectivity in Up and Down states, spiketriggered averages of whisker speed were constructed separately for Up state spikes (that is, for spikes for which the neuron was in the Up state 15-50 ms before spike generation; see Materials and Methods) and for Down state spikes (i.e., spikes for which the neuron was in the Down state 15-50 ms before spike generation). Although the Up and Down state spike-triggered averages of whisker speed had the same general shape (Fig. 9C), in all neurons the Down state spike-triggered average had a much greater peak-to-trough extent and a more rapid acceleration before spike generation.

We identified two factors that might contribute to this difference. The first factor is increased sensitivity of neurons during the Up state to small stimuli; that is, responsiveness to continuous whisker stimuli during Up and Down states resembles the responsiveness of neurons to artificial EPSPs during Up and Down states, in that only the largest stimuli are capable of evoking spikes during the Down state, whereas smaller stimuli cause a modest increase in spike probability during the Up state and no change in spike probability during the Down state. If increased sensitivity during the Up state is an important factor accounting for the difference in mean prespike stimulus, then the stimuli preceding Down state spikes should typically resemble large-scaled copies of the kernel (spike-triggered average), whereas the stimuli preceding Up state spikes should resemble both large- and smallscaled copies of the kernel.

The second factor is reduced specificity of neuronal response during the Up state: many Up state spikes may be generated as a result of intrinsic cortical dynamics. If reduced specificity is an important factor accounting for the difference in mean prespike stimulus, then more of the stimuli preceding Up state spikes should lack a significant correlation with the kernel. To determine which of these factors contributed to the observed differences, we measured the scale of each prespike stimulus (meaning the degree to which the kernel must be stretched in amplitude to best fit the actual prespike stimulus) (Fig. 9D, $x$-axis and bottom histogram) and the correlation of each prespike stimulus with the kernel (Fig. 9D, $y$-axis and left histogram) (see supplemental Fig. 3, available at www.jneurosci.org as supplemental material).

We found, in all cells, that spikes evoked from the Up state were significantly more likely to be preceded by stimuli not significantly correlated with the kernel (Up, $82 \pm 7 \%$; Down, $72 \pm$ $12 \% ; p>0.05$, Wilcoxon signed rank test). Conversely, spikes evoked from the Down state were significantly more likely to be preceded by stimuli significantly positively correlated with the kernel (Up, $15 \pm 7 \%$; Down, $27 \pm 12 \%$; $p<0.05$, Wilcoxon signed rank test). No difference between Up and Down states was observed in the proportion of spikes preceded by stimuli significantly anticorrelated with the kernel (Up, $3 \pm 2 \%$; Down, $2 \pm$ $3 \% ; p=0.63$, Wilcoxon signed rank test). In addition, among spikes preceded by stimuli significantly correlated with the kernel, spikes evoked from the Up state were more likely to follow small-scale stimuli, whereas Down state spikes were more likely to follow large-scale stimuli [percentage of significantly correlated prespike stimuli with scales less than the 30th percentile (among correlated prespike stimuli): Up state, $32 \pm 3 \%$ smallscale stimuli; Down state, $21 \pm 7 \%$; $p=0.03$, Wilcoxon signed rank test]. We conclude that both specificity and selectivity are altered during the Up state: a greater fraction of Up state spikes are preceded by stimuli that are not significantly correlated with the spike-triggered average of preceding whisker speed than in the Down state. In addition, whereas cortical neurons appear to select for similar stimulus features in Up and Down states, during the Down state, there is a somewhat greater degree of selectivity regarding which features initiate action potentials.

An alternate quantification of the same result was obtained by measuring the effects of Up and Down states on neuronal output gain using a white-noise reverse-correlation technique (de Boer and Kuyper, 1968). We obtained a measure of stimulus quality, or how similar the actual whisker stimulus was to our estimate of the cell's preferred stimulus, at each time by convolving the estimated kernel (autocorrelation-normalized spike-triggered whisker speed) against the actual pattern of whisker speeds (Fig. 10 A). We then classified each time point as Up or Down, depending on whether spikes evoked at that time would be classified as Up or Down state spikes, and constructed input-output curves relating this likeness measurement to the rate of spike output (Fig. 10B) (see Materials and Methods). We found that, compared with the Down state, the Up state was associated with a significantly greater modulation in spike rate between most- and least-like stimuli (that is, between patterns of whisker speed that most and least strongly resembled the spike-triggered average). The amount of spike rate modulation ranged from 0.01 to $0.04 \mathrm{~Hz}$ in the Down state and from 0.04 to $0.1 \mathrm{~Hz}$ in the Up state; on average, the Up state increased spike rate modulation by $127 \pm$ $37 \%(n=5$ cells; $p<0.05, t$ test) (Fig. $10 B, C)$. This finding indicates that the gain of the neuron (the amount by which it changes its output in response to changes in the input) is greater during the Up state than during the Down state. However, the Up state also exhibited a significantly greater rate of spike output in response to the worst stimuli (Up, 0.002-0.1 Hz; Down, 0-0.03 $\mathrm{Hz} ; p<0.05, t$ test). Expressed as a percentage change, therefore, the proportion by which the cell's spike rate was modulated by stimulus quality was significantly larger in the Down state than in the Up state (Up, $52 \pm 23 \%$; Down, $78 \pm 19 \% ; n=5$ cells; $p<$ $0.05, t$ test).

\section{Up states increase representation of a fluctuating stimulus}

We have seen that, during prolonged whisker stimulation, the Up state increases the responsiveness of neurons to whisker movement, while decreasing the precision of the relationship between stimulus features and timing of the resulting action potentials. We therefore hypothesized that the Up state might increase the correlation between whisker speed and spike output both by increasing the number of spikes available for stimulus representation and by altering the timing of action potential generation. To test this hypothesis, we stimulated cortical neurons with periodic, repeating patterns of whisker movement (Fig. 11). We then separated the recordings into Up and Down states, as described in Materials and Methods. The majority of action potentials were generated from the Up state (Fig. 11A). Nonetheless, and despite the weakness of the relationship (measured in Fig. 10) between stimulus input and spike output in the Down state, the spike rate histograms generated from Down state spikes were strongly regular, containing few, sharp peaks (Fig. $11 B c$ ). The Up state spike rate histograms, by comparison, contained a larger number of 
A

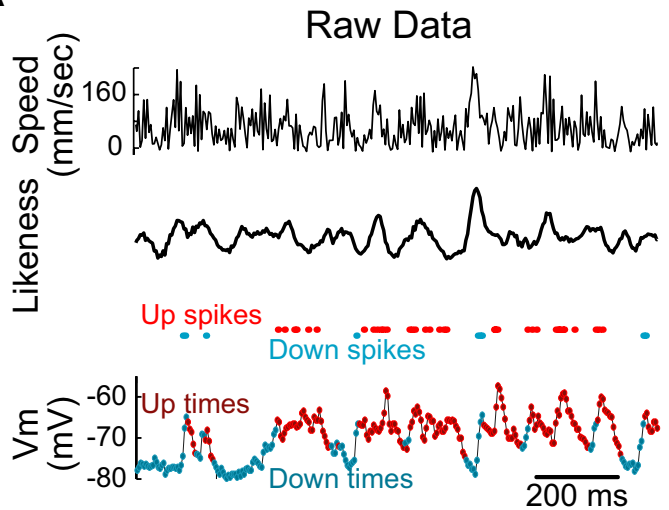

B

a

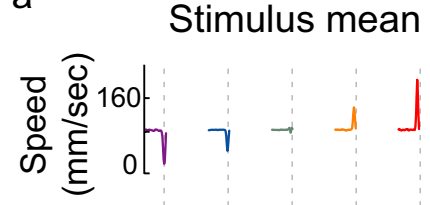

b

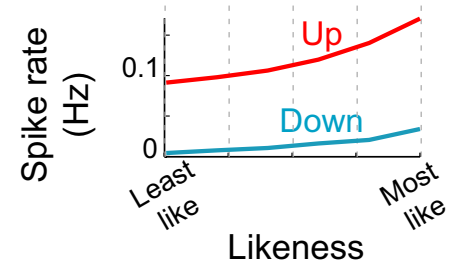

C
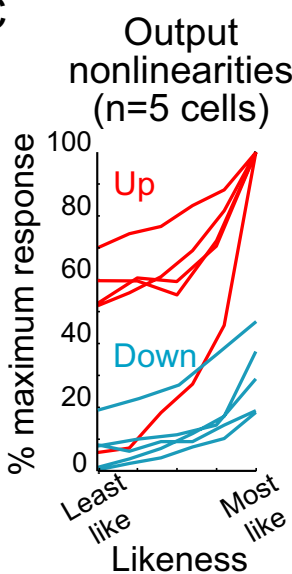

Figure 10. Up states increase neuronal gain during continuous whisker stimulation. $\boldsymbol{A}$, Whisker speed (top) was convolved with the STRF (see Fig. 9) to construct a measure of how similar the recent stimulus history was to the autocorrelation-normalized average prespike stimulus, as a function of time (likeness, middle). Each time point of the recording was sorted into Up or Down, based on how a spike would be classified if it were to occur at that time point (classification based on median-filtered membrane potential, shown at bottom; see Materials and Methods). Ba, Input- output curves were constructed by sorting time points according to state (Up vs Down) and into five levels of similarity of the prespike stimulus to the overall mean prespike stimulus ("Least like" to "Most like"); averages of the five prespike stimulus categories are shown. $\boldsymbol{B} \boldsymbol{b}$, Plotting the spike rate for each network state (Up, red; Down, blue) as a function of stimulus quality revealed both a greater baseline spike rate during the Up state and a greater modulation in spike rate by stimulus quality during the Up state. C, Overlays of normalized input-output relationships for $n=5$ cells (normalized to maximum output rate) reveal that the Up state both increased baseline spike rates and increased response gain (measured as the change in spike rate as stimulus quality changed from worst to best; $p<0.05, t$ test). However, gain, measured as the percentage change in spike rate over baseline, was significantly greater for the Down state ( $n=5$ cells; $p<0.05, t$ test), owing in large part to the low baseline firing rate of the Down state.

spikes in a greater number of peaks (Fig. $11 B d$ ). In addition, the variability of spike timing was increased in the Up state. Interestingly, the timing of the largest peaks in the Up and Down states was similar, consistent with our previous finding that the neuron was responding to similar features of whisker stimulation in both states. However, the peaks of activity in the Up state were considerably broader than during the Down state. Consistent with this, the Up state was associated with a significant increase in the peak Pearson's correlation between the speed of whisker movement and the spike output histogram (28\% increase; $p=0.03$, Wilcoxon signed rank test; $n=6$ cells; data not shown). More importantly, the Up state was associated with a significant increase in the peak Pearson's correlation between the likeness of whisker speed to the neuronal STRF and the spike output histogram

A
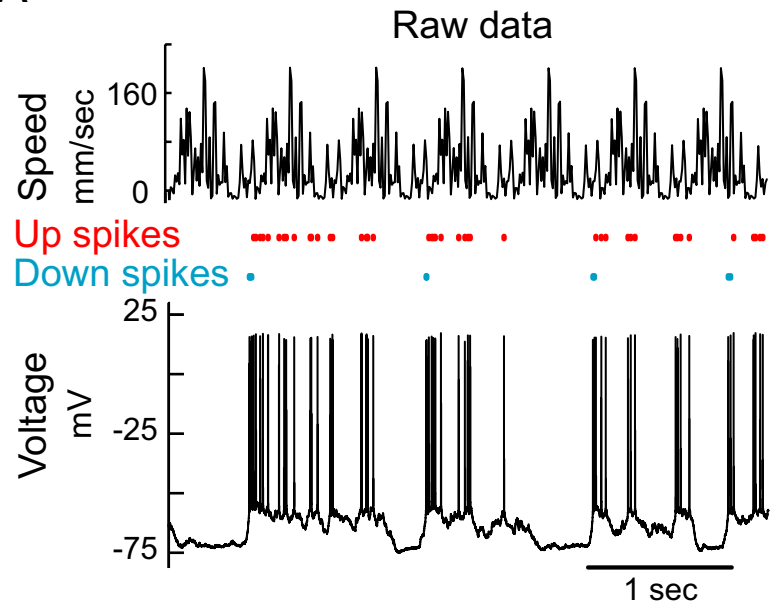

B

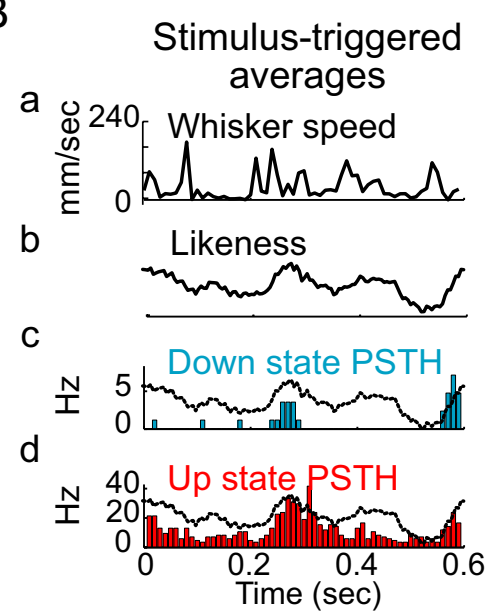

\section{Average correlations $\mathrm{n}=6$ cells}

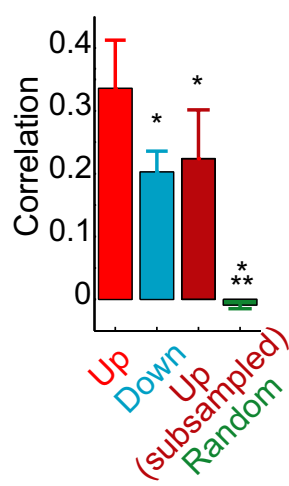

Figure 11. Up states increase the correlation between the spike response and whisker speed. $A$, Example of whisker speed during repeated presentation of the same patterned movement (top) and the resulting raw spike output and membrane potential (bottom). $\boldsymbol{B}$, Spike histograms during the Up ( $\boldsymbol{d}$, red) and Down ( $\boldsymbol{c}$, blue) states in response to the repeating pattern of whisker stimulation $(\boldsymbol{a})$. Plots are aligned to the onset of the repeating pattern of whisker stimulation. Plotted in $\boldsymbol{B} \boldsymbol{b}$ and overlain on $\boldsymbol{B} \boldsymbol{c}$ and $\boldsymbol{B} \boldsymbol{d}$ is the likeness, whisker speed convolved with the autocorrelation-corrected average prespike whisker speed (STRF). Note that rises and falls in the resemblance of the stimulus to the cell's STRF are tracked by rises and falls in the neuron's output. C, Group statistics showing that, compared with Down states (blue), Up states (red) significantly increase the peak correlation between stimulus likeness and the neuron's spike output. Subsampling the Up state PSTHs to contain the same number of spikes as the Down state PSTHs (dark red) results in a mean correlation that is not significantly different from the Down state mean correlation and that is significantly less than the mean Up state correlation. Constructing PSTHs by randomly shuffling the ISIs (green) yields a mean correlation that is significantly smaller than the Up, Down, or subsampled-Up correlations. Bar height represents mean correlation ( $n=6$ cells), and error bars represent SEs of the mean. ${ }^{*} p<0.05$; ${ }^{* *} p<$ 0.001 .

[112\%, from $0.16 \pm 0.04$ (Down) to $0.34 \pm 0.08(\mathrm{Up}) ; p<0.01$, Wilcoxon signed rank test; $n=6$ cells] (Fig. $11 C$ ). The majority of this effect was associated with the increased number of spikes available for stimulus representation during the Up state, because randomly resampling the Up state PSTHs to contain the same number of spikes as the Down state PSTHs reduced the peak correlation to $0.22 \pm 0.08$ (not significantly different from the Down state correlations). In all cases, correlations were significantly above chance, as the peak correlation obtained from constructing PSTHs by randomly sampling the interspike interval 
(ISI) distribution was $0.02 \pm 0.01$. We conclude that the output of cortical neurons in the absence of network activity is sparser and more precisely related to whisker stimulation, whereas the output of cortical neurons during elevated network activity is denser and provides a better analog representation of a continuous whisker movement.

\section{Discussion}

\section{Discrete stimulation}

We show here that although cortical network activity strongly depolarizes cortical neurons, it paradoxically decreases their responsiveness to brief, intermittent whisker stimulation (Fig. 3) (Petersen et al., 2003; Sachdev et al., 2004). Is the mechanism of this reduction in responsiveness intrinsic to the neuron recorded, or is it a network-level phenomenon? That is, are cortical neurons simply less responsive to any given excitatory synaptic conductance during the Up state, because of saturation in output rate, decrease in input resistance, excessive inhibitory tone, increases in action potential threshold, or changes in the driving force on synaptic potentials? Or, alternatively, do the stimulus-evoked synaptic conductances that cortical neurons receive change as a function of network state?

Up states were found to increase, rather than decrease, the responsiveness (both raw and baseline subtracted) of cortical neurons to synthetic EPSPs, thus demonstrating that output rate saturation, decreased input resistance, and increased inhibitory tone are insufficient to counteract the powerful facilitation of neuronal responsiveness by depolarization during the Up state (Fig. 4) (Shu et al., 2003b). Furthermore, artificial depolarization during the Down state (to mimic the Up state) dramatically increases responsiveness to brief whisker stimulation (Fig. 5). This result contradicts previous suggestions that the depolarization associated with Up states diminishes neuronal responsiveness primarily by increasing spike threshold (Sachdev et al., 2004) or by increasing the driving force on synaptic inhibition and decreasing the driving force on synaptic excitation. Although we observed an increase in spike threshold during Up states, the increase was more modest than has been previously reported and by itself was not associated with a reduction in neuronal responsiveness. Together, these findings indicate that Up states substantially increase, rather than decrease, the intrinsic excitability of cortical neurons.

\section{Interactions between cortical state and whisker stimulation}

Our measurements of the stimulus-evoked conductances imply, rather, that the reduction by Up states of neuronal responsiveness to whisker stimulation is primarily network mediated and is effected both by a reduction in the overall amount of synaptic conductance recruited by the stimulus and by an increase in the proportion of inhibition, compared with excitation, in the stimulus-evoked PSP (Fig. 8). To explain the state dependence of the amount of conductance evoked, we hypothesize that rodent somatosensory cortical and thalamocortical networks exhibit polysynaptic, recurrent interactions that permit "explosive" responses to be initiated by a sensory stimulus during the Down, but less so during the Up, state. The most dramatic example of this state dependence is demonstrated in Figures 6 and 7: transient whisker stimulation often evoked long-lasting changes in the level of local network activity and could switch the network from the Down to the Up state and vice versa. Because of the dramatic differences in neuronal response properties between Up and Down states, such long-lasting activity changes could provide a powerful mechanism by which cortical networks might rapidly modulate their own responsiveness. In addition, the response of cortical neurons to brief whisker stimuli systematically varied even within states. Over the course of the Up and Down states, the spike response to a brief whisker stimulus decreased and increased, respectively, giving rise to a slow cyclical change in responsiveness, even in the absence of changes in membrane potential (Fig. 7) (Haslinger et al., 2006). This cyclical change is related at least in part to the refractory period of the slow oscillation (Sanchez-Vives and McCormick, 2000; Shu et al., 2003a), because there is a similar cyclical change in the probability of whisker stimulation evoking state transitions (Fig. 7Cc).

\section{Mechanisms of cyclical changes in responsiveness}

Network responsiveness after the cessation of an Up state may be reduced through use-dependent depression of recurrent cortical connections (Crochet et al., 2004; Crochet and Petersen, 2006; Reig et al., 2006), the buildup of a slow afterhyperpolarization (Sanchez-Vives and McCormick, 2000; McCormick et al., 2003; Shu et al., 2003a), reduction in synaptic potentials through activity-dependent decreases in extracellular $\left[\mathrm{Ca}^{2+}\right]$ (Crochet and Petersen, 2006), or the slow activation or inactivation of a voltage-dependent inhibitory conductance (Bal and McCormick, 1996; Compte et al., 2003). The disproportionately increased excitability of fast-spiking inhibitory interneurons during the Up state may also result in reduced recurrent network activity, by enhancing the inhibitory contribution to whisker-evoked responses and consequently reducing the ability of excitatory inputs to initiate action potentials. Increased corticothalamic feedback during the Up state may also shift the thalamus out of the burst firing mode, resulting in a decrease in the number or frequency of spikes initiated in thalamocortical neurons by the whisker movement (McCormick and von Krosigk, 1992; Weyand et al., 2001) [see, however, Haslinger et al., (2006)]. Finally, tonic activity during the Up state may reduce responses to brief whisker stimuli through synaptic depression at the thalamocortical synapse (Gil et al., 1997; Beierlein and Connors, 2002; CastroAlamancos and Oldford, 2002; Hirata and Castro-Alamancos, 2006; Reig et al., 2006).

\section{Mechanisms of increased inhibition}

We additionally hypothesize that the proportionally greater inhibitory content of the whisker-evoked synaptic conductance is attributable to a greater enhancement in the responsiveness of fast-spiking GABAergic neurons than of pyramidal cells to synaptic inputs by the Up state, as we have shown previously in vitro (Shu et al., 2003a). Thalamic inputs can powerfully activate cortical interneurons (Porter et al., 2001; Swadlow and Gusev, 2002; Cruikshank et al., 2007). Although it is not yet known whether the strength of this thalamic activation is state dependent, because fast-spiking interneurons, but not pyramidal neurons, have relatively brief refractory periods and relatively linear, nonsaturating input-output relationships, the depolarization of the Up state permits fast-spiking inhibitory neurons, but not pyramidal neurons, to reliably generate several action potentials in response to a brief input, thus causing a negative shift in the reversal potential of the PSP evoked in pyramidal cells (Shu et al., 2003a; Hasenstaub et al., 2005; Cruikshank et al., 2007). In addition, fast-spiking inhibitory interneurons exhibit less spike-frequency adaptation than do pyramidal cells (McCormick et al., 1985; Nowak et al., 2003), and therefore prolonged activation of intracortical networks may shift the balance of excitation and inhibition toward inhibition. We conclude that the reduction by Up states of neuronal responsiveness to whisker stimulation is pri- 
marily network mediated (although the relative contributions of cortical and subcortical networks are not known) and is effected both by a reduction in the overall amount of synaptic conductance recruited by the stimulus and by an increase in the proportion of inhibition in the stimulus-evoked PSP (Fig. 8).

\section{Continuous stimulation}

During natural exploration and behavior, rodent whiskers often receive prolonged stimulation (Prigg et al., 2002; Knutsen et al., 2006), and the response properties of somatosensory cortical neurons to such stimulation are markedly different from those associated with brief, punctuate stimuli (Armstrong-James and Fox, 1987; Kleinfeld et al., 2002; Arabzadeh et al., 2004; Knutsen et al., 2006; Melzer et al., 2006). When we examined the effects of changes in cortical network activity on responses to continuous whisker stimulation, we found that cortical networks continued to alternate between Up-like states (i.e., periods of increased multiple-unit activity simultaneous with tonic intracellular depolarization and lasting 200-1000 ms) and Down-like states (i.e., periods of decreased multiple-unit activity with tonic intracellular hyperpolarization) (supplemental Fig. 2, available at www.jneurosci.org as supplemental material). Up-like states were found to increase both the overall level of spike output after a stimulus and the neurons' gain, or change in spike output resulting from a change in input quality (Figs. 9, 10). We additionally found that, despite the decrease in neuronal sensitivity to intermittent whisker stimulation during the Up state, the Up state was nonetheless associated with an increase in the correlation between neurons' spike response and the variations in whisker speed during ongoing whisker movements (Fig. 11). Related investigations of the spike-triggered average of whisker velocity or speed and reconstructions of the output nonlinearity have been performed in first-order (trigeminal ganglion) neurons of the whisker system, as well as on multiple-unit cortical recordings (Jones et al., 2004; Arabzadeh et al., 2005). These previous investigations have demonstrated that, whereas the first-order neurons respond faithfully and reliably to specific stimulus features, cortical neurons fire with lower fidelity and at a greater range of times during a stimulus. Here, we show that this variability in the timing of spike output is particularly associated with increases in cortical background activity.

\section{Specificity versus sensitivity}

During continuous whisker stimulation, most (70-90\%) of the spikes evoked from both Up and Down states were not clearly associated with whisker stimulation, either as determined from examining the significance of the linear correlation between the prespike stimulus and the estimated kernel (Fig. 9) or as determined by examining the rate at which spikes were generated after stimuli that were anticorrelated with the kernel (Fig. 10). However, a greater proportion of Down state spikes were significantly associated with the stimulus, and among Down state spikes that were likely stimulus-evoked, a greater proportion were generated after stimuli that were large-scale copies of the speed kernel (that is, after the best stimulus features). During repeating patterned whisker stimulation, Down state spikes were mostly generated at stereotyped times during the stimulus, times at which the stimulus likeness was large. Thus, during Down states, neurons appear to be acting more as threshold detectors or feature detectors, elevating their firing rate preferentially in response to the best stimuli. In contrast, during the Up state, although more stimulus-irrelevant spikes were generated, a much greater number of stimulus-related spikes were generated as well (Figs. 9, 10), and a greater proportion of those spikes were generated after weaker stimulus features. During repeating whisker stimulation, Up state spikes were generated at a variety of times during the stimulus, both after strong- and weak-likeness stimuli: the output PSTH was a closer linear or analog representation of stimulus likeness. Thus, during Up states, neurons appear to be transmitting more of an analog representation of stimulus quality. We conclude that, in the absence of network activity, cortical responses to continuous whisker stimulation are relatively sparse and specific, whereas in the presence of network activity, cortical neurons are more sensitive to weak stimulus features and respond more strongly and more linearly.

\section{Functional consequences of background activity}

In the absence of synaptic inputs, cortical neurons are typically $10-30 \mathrm{mV}$ removed from firing threshold. If, in order for a cortical neuron to generate an action potential in response to sensory or synaptic input, it were necessary to depolarize the neuron anew from its resting membrane potential to firing threshold for each stimulus, then only infrequent whisker contacts, or contacts delivered during states of minimal preadaptation, would have a chance of evoking cortical action potentials. However, the ability of cortical networks to control their own excitability through recurrent activity permits the cortex to maintain its neurons near firing threshold, through depolarizations generated by the loosely synchronous coactivation of the thousands of recurrently connected neurons in the local cortical network (Steriade et al., 1993, 2001; Shu et al., 2003b). The interaction between stimuli and subsequent cortical state might permit cortical networks to self-adapt their responsiveness to stimulus statistics, permitting responsiveness even when the feedforward sensory input is weak, or, through feedback to subcortical networks, to control the strength of feedforward sensory information they receive. Locally generated barrages of synaptic activity could increase the excitability of, and permit sensory transmission through, limited regions of cortex, regulating the central flow of sensory information. Additional investigation of the interactions between sensory stimulation and cortical circuitry will undoubtedly yield a better understanding of the dynamic control of sensory responsiveness within the cerebral cortex.

\section{References}

Arabzadeh E, Panzeri S, Diamond ME (2004) Whisker vibration information carried by rat barrel cortex neurons. J Neurosci 24:6011-6020.

Arabzadeh E, Zorzin E, Diamond ME (2005) Neuronal encoding of texture in the whisker sensory pathway. PLoS Biol 3:e17.

Arieli A, Shoham D, Hildesheim R, Grinvald A (1995) Coherent spatiotemporal patterns of ongoing activity revealed by real-time optical imaging coupled with single-unit recording in the cat visual cortex. J Neurophysiol 73:2072-2093.

Armstrong-James M, Fox K (1987) Spatiotemporal convergence and divergence in the rat S1 "barrel" cortex. J Comp Neurol 263:265-281.

Azouz R, Gray CM (1999) Cellular mechanisms contributing to response variability of cortical neurons in vivo. J Neurosci 19:2209-2223.

Bal T, McCormick DA (1996) What stops synchronized thalamocortical oscillations? Neuron 17:297-308.

Beierlein M, Connors BW (2002) Short-term dynamics of thalamocortical and intracortical synapses onto layer 6 neurons in neocortex. J Neurophysiol 88:1924-1932.

Borg-Graham LJ, Monier C, Fregnac Y (1998) Visual input evokes transient and strong shunting inhibition in visual cortical neurons. Nature 393:369-373.

Cahalan MD, Almers W (1979) Interactions between quaternary lidocaine, the sodium channel gates, and tetrodotoxin. Biophys J 27:39-55.

Castro-Alamancos MA, Oldford E (2002) Cortical sensory suppression during arousal is due to the activity-dependent depression of thalamocortical synapses. J Physiol (Lond) 541:319-331.

Compte A, Sanchez-Vives MV, McCormick DA, Wang XJ (2003) Cellular and network mechanisms of slow oscillatory activity $(<1 \mathrm{~Hz})$ and wave propagations in a cortical network model. J Neurophysiol 89:2707-2725. Cowan RL, Wilson CJ (1994) Spontaneous firing patterns and axonal pro- 
jections of single corticostriatal neurons in the rat medial agranular cortex. J Neurophysiol 71:17-32.

Crochet S, Petersen CC (2006) Correlating whisker behavior with membrane potential in barrel cortex of awake mice. Nat Neurosci 9:608-610.

Crochet S, Fuentealba P, Timofeev I, Steriade M (2004) Selective amplification of neocortical neuronal output by fast prepotentials in vivo. Cereb Cortex 14:1110-1121.

Cruikshank SJ, Lewis TJ, Connors BW (2007) Synaptic basis for intense thalamocortical activation of feedforward inhibitory cells in neocortex. Nat Neurosci 10:462-468.

de Boer R, Kuyper P (1968) Triggered correlation. IEEE Trans Biomed Eng 15:169-179.

Dorval AD, Christini DJ, White JA (2001) Real-Time Linux dynamic clamp: a fast and flexible way to construct virtual ion channels in living cells. Ann Biomed Eng 29:897-907.

Fellous JM, Rudolph M, Destexhe A, Sejnowski TJ (2003) Synaptic background noise controls the input/output characteristics of single cells in an in vitro model of in vivo activity. Neuroscience 122:811-829.

Gil Z, Connors BW, Amitai Y (1997) Differential regulation of neocortical synapses by neuromodulators and activity. Neuron 19:679-686.

Haider B, Duque A, Hasenstaub AR, McCormick DA (2006) Neocortical network activity in vivo is generated through a dynamic balance of excitation and inhibition. J Neurosci 26:4535-4545.

Hartigan JA, Hartigan PM (1986) The dip test of unimodality. Ann Statist 13:70-84.

Hartmann MJ, Johnson NJ, Towal RB, Assad C (2003) Mechanical characteristics of rat vibrissae: resonant frequencies and damping in isolated whiskers and in the awake behaving animal. J Neurosci 23:6510-6519.

Hasenstaub A, Shu Y, Haider B, Kraushaar U, Duque A, McCormick DA (2005) Inhibitory postsynaptic potentials carry synchronized frequency information in active cortical networks. Neuron 47:423-435.

Haslinger R, Ulbert I, Moore CI, Brown EN, Devor A (2006) Analysis of LFP phase predicts sensory response of barrel cortex. J Neurophysiol 96:1658-1663.

Hipp J, Arabzadeh E, Zorzin E, Conradt J, Kayser C, Diamond ME, Konig P (2006) Texture signals in whisker vibrations. J Neurophysiol 95:1792-1799.

Hirata A, Castro-Alamancos MA (2006) Relief of synaptic depression produces long-term enhancement in thalamocortical networks. J Neurophysiol 95:2479-2491.

Jones LM, Lee S, Trageser JC, Simons DJ, Keller A (2004) Precise temporal responses in whisker trigeminal neurons. J Neurophysiol 92:665-668.

Kleinfeld D, Sachdev RN, Merchant LM, Jarvis MR, Ebner FF (2002) Adaptive filtering of vibrissa input in motor cortex of rat. Neuron 34:1021-1034.

Knutsen PM, Pietr M, Ahissar E (2006) Haptic object localization in the vibrissal system: behavior and performance. J Neurosci 26:8451-8464.

McCormick DA, von Krosigk M (1992) Corticothalamic activation modulates thalamic firing through glutamate "metabotropic" receptors. Proc Natl Acad Sci USA 89:2774-2778.

McCormick DA, Connors BW, Lighthall JW, Prince DA (1985) Comparative electrophysiology of pyramidal and sparsely spiny stellate neurons of the neocortex. J Neurophysiol 54:782-806.

McCormick DA, Shu Y, Hasenstaub A, Sanchez-Vives M, Badoual M, Bal T (2003) Persistent cortical activity: mechanisms of generation and effects on neuronal excitability. Cereb Cortex 13:1219-1231.

Melzer P, Sachdev RN, Jenkinson N, Ebner FF (2006) Stimulus frequency processing in awake rat barrel cortex. J Neurosci 26:12198-12205.

Metherate R, Ashe JH (1993) Ionic flux contributions to neocortical slow waves and nucleus basalis-mediated activation: whole-cell recordings in vivo. J Neurosci 13:5312-5323.

Nathan T, Jensen MS, Lambert JD (1990) The slow inhibitory postsynaptic potential in rat hippocampal CA1 neurones is blocked by intracellular injection of QX-314. Neurosci Lett 110:309-313.

Neimark MA, Andermann ML, Hopfield JJ, Moore CI (2003) Vibrissa resonance as a transduction mechanism for tactile encoding. J Neurosci 23:6499-6509.

Nowak LG, Azouz R, Sanchez-Vives MV, Gray CM, McCormick DA (2003) Electrophysiological classes of cat primary visual cortical neurons in vivo as revealed by quantitative analyses. J Neurophysiol 89:1541-1566.

Pare D, Shink E, Gaudreau H, Destexhe A, Lang EJ (1998) Impact of spon- taneous synaptic activity on the resting properties of cat neocortical pyramidal neurons in vivo. J Neurophysiol 79:1450-1460.

Perkins KL, Wong RK (1995) Intracellular QX-314 blocks the hyperpolarization-activated inward current Iq in hippocampal CA1 pyramidal cells. J Neurophysiol 73:911-915.

Petersen CC, Hahn TT, Mehta M, Grinvald A, Sakmann B (2003) Interaction of sensory responses with spontaneous depolarization in layer $2 / 3$ barrel cortex. Proc Natl Acad Sci USA 100:13638-13643.

Pinto DJ, Brumberg JC, Simons DJ (2000) Circuit dynamics and coding strategies in rodent somatosensory cortex. J Neurophysiol 83:1158-1166.

Porter JT, Johnson CK, Agmon A (2001) Diverse types of interneurons generate thalamus-evoked feedforward inhibition in the mouse barrel cortex. J Neurosci 21:2699-2710.

Powers RL, Arnett DW (1981) Spatio-temporal cross-correlation analysis of catfish retinal neurons. Biol Cybern 41:179-196.

Prigg T, Goldreich D, Carvell GE, Simons DJ (2002) Texture discrimination and unit recordings in the rat whisker/barrel system. Physiol Behav 77:671-675.

Reig R, Gallego R, Nowak LG, Sanchez-Vives MV (2006) Impact of cortical network activity on short-term synaptic depression. Cereb Cortex 16:688-695.

Ringach DL, Hawken MJ, Shapley R (2002) Receptive field structure of neurons in monkey primary visual cortex revealed by stimulation with natural image sequences. J Vis 2:12-24.

Sachdev RN, Sellien H, Ebner F (2001) Temporal organization of multiwhisker contact in rats. Somatosens Mot Res 18:91-100.

Sachdev RN, Sato T, Ebner FF (2002) Divergent movement of adjacent whiskers. J Neurophysiol 87:1440-1448.

Sachdev RN, Ebner FF, Wilson CJ (2004) Effect of subthreshold up and down states on the whisker-evoked response in somatosensory cortex. J Neurophysiol 92:3511-3521.

Sanchez-Vives MV, McCormick DA (2000) Cellular and network mechanisms of rhythmic recurrent activity in neocortex. Nat Neurosci 3:1027-1034.

Sharp AA, O’Neil MB, Abbott LF, Marder E (1993) The dynamic clamp: artificial conductances in biological neurons. Trends Neurosci 16:389-394.

Shoykhet M, Doherty D, Simons DJ (2000) Coding of deflection velocity and amplitude by whisker primary afferent neurons: implications for higher level processing. Somatosens Mot Res 17:171-180.

Shu Y, Hasenstaub A, McCormick DA (2003a) Turning on and off recurrent balanced cortical activity. Nature 423:288-293.

Shu Y, Hasenstaub A, Badoual M, Bal T, McCormick DA (2003b) Barrages of synaptic activity control the gain and sensitivity of cortical neurons. J Neurosci 23:10388-10401.

Shu Y, Duque A, Yu Y, Haider B, McCormick DA (2007) Properties of action-potential initiation in neocortical pyramidal cells: evidence from whole cell axon recordings. J Neurophysiol 97:746-760.

Stafstrom CE, Schwindt PC, Chubb MC, Crill WE (1985) Properties of persistent sodium conductance and calcium conductance of layer $\mathrm{V}$ neurons from cat sensorimotor cortex in vitro. J Neurophysiol 53:153-170.

Steriade M, Nunez A, Amzica F (1993) A novel slow ( $<1 \mathrm{~Hz}$ ) oscillation of neocortical neurons in vivo: depolarizing and hyperpolarizing components. J Neurosci 13:3252-3265.

Steriade M, Timofeev I, Grenier F (2001) Natural waking and sleep states: a view from inside neocortical neurons. J Neurophysiol 85:1969-1985.

Swadlow HA, Gusev AG (2002) Receptive-field construction in cortical inhibitory interneurons. Nat Neurosci 5:403-404.

Theunissen F, Miller JP (1995) Temporal encoding in nervous systems: a rigorous definition. J Comput Neurosci 2:149-162.

Timofeev I, Contreras D, Steriade M (1996) Synaptic responsiveness of cortical and thalamic neurones during various phases of slow sleep oscillation in cat. J Physiol (Lond) 494:265-278.

Uhlenbeck GE, Ornstein LS (1930) On the theory of Brownian motion. Phys Rev 36:823-841.

Weyand TG, Boudreaux M, Guido W (2001) Burst and tonic response modes in thalamic neurons during sleep and wakefulness. J Neurophysiol 85:1107-1118.

Yeh JZ (1978) Sodium inactivation mechanism modulates QX-314 block of sodium channels in squid axons. Biophys J 24:569-574. 